\title{
Dynamic boundary conditions for a coupled convection-diffusion model with heat effects : applications in cross-contamination control
}

\author{
N. Santatriniaina ${ }^{1 *}$, J. Deseure ${ }^{2}$, T.Q. Nguyen ${ }^{3}$, H. Fontaine ${ }^{3}$, C. Beitia ${ }^{3}$, L. Rakotomanana ${ }^{1}$ \\ ${ }^{1}$ Mathematical Research Institute of Rennes, IRMAR, UMR 6625, CNRS, 263, Av. du Général Leclerc, \\ University of Rennes, 35042, Rennes, France \\ ${ }^{2}$ Laboratoire d'Electrochimie et de Physico-Chimie des Matériaux et des Interfaces, LEPMI, UMR 5279, CNRS, \\ 1130, rue de la piscine, 38402, Grenoble, France \\ 3 Commissariat à l'Energie Atomique: CEA/LETI, 17, rue des Martyrs, 38054, Grenoble Cedex 9, France \\ *Corresponding author E-mail: nirinasantatriniaina@gmail.com
}

Copyright (C2015 N. Santatriniaina et. al. This is an open access article distributed under the Creative Commons Attribution License, which permits unrestricted use, distribution, and reproduction in any medium, provided the original work is properly cited.

\begin{abstract}
This work investigates the mass transfer of the Airborne Molecular cross Contamination (AMCs) between the Front Opening Unified Pod (FOUP) and wafer (silicon substrates) during the microelectronics devices manufacturing using dynamic boundary conditions. Such cross-contamination phenomena lead to detrimental impact on production yield in microelectronic industry and a predictive approach using modelling and computational methods is a very strong way to understand and qualify the AMCs cross contamination processes. The FOUP is made of polymeric materials and it is considered as a heterogeneous porous media, it can asorb and desorb the contaminant, thus the modelled processes are the contamination of two-component in transient flow. Coupled diffusion and convection-diffusion model with heat effects are used to define the phenomena. The present methodology is, first using the optimization methods with the numerical solution in order to define the physical constants of various materials which have been studied experimentally and separately, and the second using the finite element methods including these physical constants and relevant interface condition in order to take into account the adsorption kinetics law. Numerical methods to solve the problem are proposed. The dynamics behavior of the AMCs analysis was determined thanks to the switch of Dirichlet to Neumann condition. The mathematical model preserves the classical forms of the diffusion and convection diffusion equations and yields to consistent form of the Fick's law. The computed results are in correlation with the experimental measurements. Some numerical results are presented in this work.
\end{abstract}

Keywords: Cross-contamination, Coupling technique, dynamic boundary conditions, finite element methods, mathematical model.

\section{Introduction}

In the semiconductor manufacturing, the particle, bacterian, metallic and molecular contamination of the wafer are a crucial subjects. These contaminations can potentially cause defects in devices performance. The wafer carrier and storage play an important role for contamination control [1]-[2]. The Front Unified Pods (FOUPs) is used to transport wafers from one point to another point and to protect the wafer with contamination [2]- [3]. The use of 
these pods may occurs new kinds of contamination the airborne molecular contamination [3]-[4]. The wafer post processed contamines the pods with volatile acids and contamines news wafer and vice-versa [4], [5]-[6]. The FOUP is made with a porous polymers materials well-known with its adsorption and outgass properties [1]-[2], [5]-[6]. These physical phenomena are traduced by sorption phenomena as adsorption and desorption [7], [8]. They are also able to absorb volatile compounds present in their atmosphere coming from the connection to an equipment or from the release of wafers post processed [1]-[4], [9]. These phenomena result a reversible and a irreversible outgassing of contaminant previously trapped in polymer [9], [10], [11].

When one object becomes contaminated by either direct or indirect contact with another object which is already contaminated, in this case we talk about cross contamination [5]-[6]. In microelectronics industry, this process takes place generally at the FOUPs who contains the wafer before and after fabrication process [2], [4]. Some fabrication method is the source of contamination, when the wafer is already processed its surface is contamined by the volatile acids [1]. The wafer is the exposed to the FOUP's atmosphere and a volatile acid contamination may happened from wafer to FOUP [2]. When the wafer is removed or the FOUP is opened, a cross-contamination may happened, the contaminant moves from FOUP to new wafer [4], [5].

To ensure the integrated circuit manufacturing, especially through the utilisation of $300 \mathrm{~mm}$ wafer manufacturing technologies, new methods are needed for systematic characterization, the numerical method for analysis and control of the underlying system and processes behaviour [5]-[6]. The modelling and computational method is excellent method to predict and quantify a physical phenomena as AMCs cross contamination in FOUP [6]-[7]. Numerous method are already develop to characterize by using experimental process to quantify these phenomena. J. Crank and al. established semi-analytical solution of the diffusion equation and with many cases [12], [14]. Some papers deals with kinetics adsorption and deposition the contamination process with volatile acid in the pods [15]-[17]. A finite element simulation of the purge method and numerous method of characterization are also developed in [3], [18], [19]. Another paper studies the kinetics of sorption and the decontamination process characterization [20], $[21],[22]$.

This work describe and develop a mathematical model and an appropriate numerical tools using the finite element formulation for the dynamic of the airborne molecular cross-contamination in microelectronic industry. The model includes the heat effects by using the Arrhenius law and the heat equation. We use the diffusion and convection/diffusion model with/without heat effects to describe the phenomena. A dynamic boundary and interface conditions are applied to simulate the industrial conditions of the pods. Numerical approximations are presented to solve the problem. The validation of the model is based on the correlation study between the observed datas (experimental measurement) and the computed results with the model.

\section{Physical problem and experiment procedure}

\subsection{FOUP's geometry}

Thi Quynh Nguyen [1], Paola G. [2] and al. established new experimental protocol to quantify the cross-contaminantion for each material constituent of the FOUP in order to choice the optimal material versus contaminant. The utilization of the FOUP in the microelectronic industry are defined in several step as opening, closing and cleaning (purge, outgassing, waiting) steps for the characterisation [1], [5], [6]. Let us add in this main steps an other step, an intentionnal contamination event, in order to understand the dynamics and to quantify the AMCs cross contamination for some contaminant vs FOUP materials types (polymer). For the simulation, we assume the following geometry given by the figure 1 .

\subsection{Physical problem and experiment procedure}

For the experimental measurement, we referred to [1], [2] and resume the experimental measurement as two processes: the characterization of the diffusion coefficient and the characterization of the AMCs cross contamination in FOUP scale. In order to characterize the diffusion coefficient, we use a chemical reactor in which we insert a thin membrane of the material constituent of the pods. An inlet supplies the reactor and outlet contaminant fluids constant flow in order to measured the adsorbed quantity of the contaminant in the polymer. The fluid contaminant is obtained by mixing air with a initial concentration (some ppbv). In general, the flow rate of the contemined airflow is $3[\mathrm{ml} / \mathrm{min}]$ (constant). In order to analyse the membrane and to quantify the sorbed concentration, we dissolve the adsorbed contaminant molecules in the polymer into the water leading to ion formation. The water has been analysed by Ionic Chromatography (IC). This method is dedicated to volatile acids. 


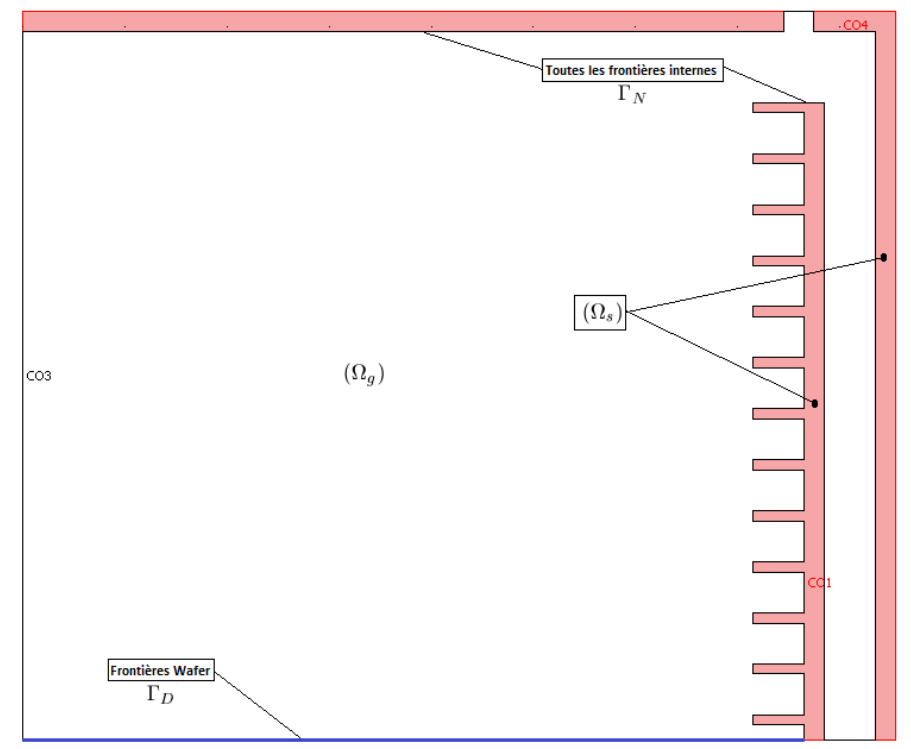

Figure 1: Quarter of the FOUP with the wafer support and the filter port (top right), 2D elementary representative volume for the simulation. $\Omega_{s}$ denotes the polymer, $\Omega_{g}$ denotes the FOUP's atmosphere, $\Gamma_{D}$ denotes the wafer surface and $\Gamma_{N}$ denotes the interface between the FOUP's atmosphere and the polymer.

For the AMC's characterization in the pods, we assume the same procedure as define above for the quantification. The FOUP's atmosphere after wafers removing has been analysed with specific technics. An intentional contamination is realised in order to create the initial concentration (this process present he contamination from the wafer). The total amount of the volatile acids concentration was monitored with an Ion Mobility Spectrometer (IMS). After wafers removing, the pods was connected by specific outlet/inlet the filter ports replacing FOUP filters to a bubbling system which is composed of two bubblers in series filled with Deionized Water (DIW). The air in the pods was pumped through bubblers to dissolve molecules into the water leading to ions formation. The water was then analysed by Ionic Chromatography. The bubbling solution was also analyzed by ICP-OES (Inductively Coupled Plasma-Optical Emission Spectrometry). The amount of acids sorbed on the FOUP surface was collected by DIW leaching, then characterized by ionic chromatography.

\section{Mathematical settings}

The modeling of the adsorption of the airborne molecular cross-contamination between wafer and FOUP is based on adsorption phenomena (physical adsorption, chemical adsorption) [5], [6]. In general, two concepts of adsorption exist, physical and chemistry adsorption models. A detailed review of various adsorption kinetics (adsorption, desorption) models was given in [12], [15]. Generally, adsorption processes can be divided into two classes, reversible and irreversible adsorption [17], [21], [22]. In this work the kinetics of adsorption between the FOUP's atmosphere and the polymer is no yet taken into account but traduced as a Henry law at the interface. The simplest model for adsorption is diffusion based by the Fick's law which consist to describe the concentration with respect the location and time as in [12]. In this model, the transport towards the surface is purely diffusive and we investigate the concentration in the internal surface of the pods by using the thermodynamics laws. This thermodynamics law is traduced by the Henrys constant. It is used to connect the concentration of the contaminant at the FOUP's atmosphere and the concentration at the internal surface of the pods.

\subsection{Nomenclature}

Let us introduce several notations which will be throughout this work. Let 


\begin{tabular}{|c|c|c|}
\hline$\nabla$. & $:$ & Divergence operator \\
\hline$\nabla$ & $:$ & Gradient operator \\
\hline$h_{0}$ & $:$ & Henry constant \\
\hline$s$ & $:$ & Represent the polymer \\
\hline$g$ & : & Represent the contaminant \\
\hline $\mathbf{u}$ & $:$ & Contaminant velocity \\
\hline $\mathbf{q}_{i}$ & $:$ & Source \\
\hline $\mathbf{q}_{1}$ & $:$ & Source in the polymer $s$ \\
\hline $\mathbf{q}_{2}$ & : & Source in the contaminant $g$ \\
\hline $\mathbf{D}_{s}$ & $:$ & Diffusion coefficient in polymer $s$ \\
\hline $\mathbf{D}_{g}$ & $:$ & Diffusion coefficient in the contaminant $g$ \\
\hline $\mathbf{D}_{0 s}$ & : & Diffusion coefficient in the polymer at the reference temperature $T$ \\
\hline $\mathbf{D}_{0 g}$ & : & Diffusion coefficient in the FOUP's atmosphere at the reference temperature $T$ \\
\hline$C^{s}$ & : & Concentration in polymer \\
\hline$C^{g}$ & : & Concentration in internal FOUP's atmosphere \\
\hline$C_{0}$ & : & Concentration on the wafer surface \\
\hline $\mathbf{n}$ & : & Outer unit normal vector \\
\hline $\mathbf{r}$ & : & Heat source \\
\hline$N_{0}^{g}$ & : & Inlet concentration flux \\
\hline$H(t-\varepsilon)$ & : & Heaviside function with delay $\varepsilon$ \\
\hline$\Omega_{s}$ & : & Polymer subdomain \\
\hline$\Omega_{g}$ & $:$ & Contaminant subdomain \\
\hline$\Gamma_{i}$ & $:$ & Denote the boundary of the domain $i$ \\
\hline$\rho^{s}$ & : & Density of the polymer \\
\hline$c^{s}$ & : & Specific heat of the polymer \\
\hline$\kappa_{s}$ & $:$ & Heat conductivity constant of the polymer \\
\hline$(*)$ & $:$ & Variable with the temperature effect using Arrhenius law \\
\hline$E$ & $:$ & Activation energy \\
\hline
\end{tabular}

\subsection{Transport equation}

Let us $\Omega_{s} \subset \mathbb{R}^{2}$ and $\Omega_{g} \subset \mathbb{R}^{2}$ be a open bounded domain for the polymer $s$ and for the contaminant $g$ respectively. The boundary $\Gamma$ is a part of $\partial \Omega:=\cup_{i} \Gamma_{i}$ and is divided in two parts for Dirichlet boundary condition on $\Gamma_{D}$ and Neumann boundary condition on $\Gamma_{N}$, in this work $\Gamma_{N}:=\partial \Omega^{s} \cap \partial \Omega^{g}\left(\partial \Omega^{s}\right.$ and $\partial \Omega^{g}$ are defined in figure 2). The time is denoted by $t \in[0, T]$. The time-range is given by $[0, T]$ with $T \in \mathbb{R}_{+}$.

The FOUP geometry is very complex and it content many singularity cf. figure 1 . In order to outcome the geometry complexity, a simplified geometry will be used to solve numerically the model. We use the same REV (Representative Elementary Volume) for the FOUP's material (support of the wafer and the body's polymer). Sometimes, this simplification is useful to enhance the performance of vector rendering or to reduce complexity of the geometry and to ensure the numerical convergence and in order to reduce the computational subdomain. This might be especially handy for a geometry a small scale. So, let us assume two rectangular geometries (control geometry), in 2-dimensions represented by the domain $\Omega_{g}$ and $\Omega_{s}$ respectively the FOUP atmosphere and polymer membrane cf. figure 2 .

For simplicity, we reduce this geometry defined in figure 1 to a problem on rectangular domain by prescribed the boundary on the fictive artificial boundary $\Gamma_{D}$ (wafer surface). We will use the same notation $\Gamma_{D}$ as defined on figure 2. $\Gamma_{N}$ is the interface between the FOUP's atmosphere and the body's polymer.

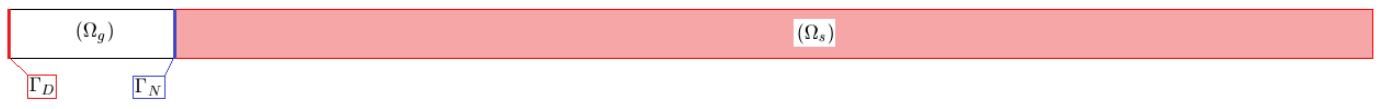

Figure 2: Simplified Geometry: the first subdomain $\Omega_{s}$ is for the contaminant and the second subdomain $\Omega_{g}$ is for the contaminant. An exact ratio for the total surface exchange are quantified in order to define the size of the elementary representative volume. $\Gamma_{D}$ respesent the wafer (source of the contaminant) and $\Gamma_{N}$ is the interface between the contaminant $g$ and the polymer $s$.

The mathematical model of diffusion process in the domain $\Omega^{g}$ and $\Omega^{s}$ are based on Fick's second law. And at the interface of these domains a kinetics law will be established. According to Fick's first and second law as in 
[5]-[6], [12], also known as the diffusion equation the flux per unit of area perpendicular to the flux direction is given by

$J_{i}=-\mathbf{D}_{i} \nabla C^{i}$

Where here $i:=s, g$.

The AMCs cross contamination model is governed by a diffusion time dependent process in which the rate of diffusion is fraction of time. In this process, the contaminant moves from a region of high concentration (wafer) to the one region low concentration (internal surface of FOUP) [5]-[6], [12]. We have respectively the following conservation equation for the species $i$ in the polymer $s$ and in the contaminant $g$ as:

$\frac{\partial C^{i}}{\partial t}=-\nabla \cdot J_{i}+\mathbf{q}_{i}$

$\frac{\partial C^{i}}{\partial t}=-\nabla \cdot J_{i}-\nabla \cdot \mathbf{u} C^{i}+\mathbf{q}_{i}$

During the outgass phenomena the contaminant moves from FOUP to the new wafer. We assume that, on $\Gamma_{D}$, we have the wafer area (wafer surface), in which the source of contamination take place during the contamination time $t_{c} \in \mathbb{R}_{+}$.

We assume that the advection parts and reaction time scale are slow compared to the diffusive time scale. The model for transient contaminant transfer between the wafer and the internal part of the FOUP is given by :

$$
\left\{\begin{array}{llr}
\frac{\partial C^{s}}{\partial t} & =\nabla \cdot\left(\mathbf{D}_{s} \nabla C^{s}\right)+\mathbf{q}_{1} & \text { in }\left(\Omega_{s} \times[0, T]\right) \\
C^{s} & =h_{0} C^{g} & \text { on }\left(\Gamma_{N}\right) \\
\mathbf{D}_{s} \nabla C^{s} \cdot \mathbf{n} & =0 & \text { on }\left(\partial \Omega_{s}-\Gamma_{N}\right) \\
\frac{\partial C^{g}}{\partial t} & =\nabla \cdot\left(\mathbf{D}_{g} \nabla C^{g}\right)-\nabla \cdot\left(\mathbf{u} C^{g}\right)+\mathbf{q}_{2} & \text { in }\left(\Omega_{g} \times[0, T]\right) \\
\left(-\mathbf{D}_{g} \nabla C^{g}+\mathbf{u} C^{g}\right) \cdot \mathbf{n} & =N_{0}^{g}+k_{c}\left[C_{0} H(t-\varepsilon)-C^{g}\right] & \text { on }\left(\Gamma_{D}\right) \\
C^{g} & =\frac{C^{s}}{h_{0}} & \text { on }\left(\Gamma_{N}\right) \\
\left(-\mathbf{D}_{g} \nabla C^{g}+\mathbf{u} C^{g}\right) \cdot \mathbf{n} & =0 & \text { on }\left(\partial \Omega_{g}-\left(\Gamma_{N} \cup \Gamma_{D}\right)\right)
\end{array}\right.
$$

\subsection{Boundary conditions}

The industrial condition of AMCs cross contamination prescribe the use of the switch condition on the boundary condition (Dirichlet-Neumann switch). During the contamination process, the wafer post processed contamines with a constant concentration $C_{0} \in \mathbb{R}_{+}$, we have a Dirichlet condition during this process. After the contamination step, these boundary condition change to Neumann boundary condition because the wafer stop to outgass contaminant on the wafer surface. In practice, this method is defined as the contaminant concentration maximum value is $C_{0} \in \mathbb{R}_{+}$during the contamination time $t_{c} \in \mathbb{R}_{+}$, and when the contamination time is finished the wafer surface is considered as a neutral surface. So we need to switch this two conditions during this time-range. In fact, the first approximation is based by an heuristic method, it consist to write the condition as a flux inflow and we use the parameter $k_{c} \in \mathbb{R}_{+}$to switch the two conditions (Dirichlet/Neumann). In this case, the boundary condition in wafer area is given by:

$$
\left(-\mathbf{D}_{g} \nabla C^{g}+\mathbf{u} C^{g}\right) \cdot \mathbf{n}=N_{0}^{g}+k_{c}\left[C_{0} H(t-\varepsilon)-C^{g}\right]
$$

We assume that $N_{0}^{g}=0$ (no initial flux) and we assume that a transient boundary conditions with a laminar gas flow on this boundary $(\mathbf{u}=0)$, when $k_{c}$ is sufficiently large, we have the Dirichlet condition i.e $C_{0} H(t-\varepsilon) \simeq C^{g}$ and if $k_{c}=0$ we have the Neumann's boundary condition (for the neutral surface of the wafer), i.e $\left(-\mathbf{D}_{g} \nabla C^{g}+\mathbf{u} C^{g}\right) \cdot \mathbf{n}=0$. Then, we need to conditionate $C_{0}$ and $k_{c}$ a parametric study was made to ensure the stability.

We have,

if $\left\{\begin{array}{lr}k_{c} \gg 1 \Rightarrow C^{g} \simeq C_{0} H(t-\varepsilon) & \text { if } 0<t \leq\left(t_{c}+t_{p}\right) \\ k_{c}=0 \Rightarrow\left(-\mathbf{D}_{g} \nabla C^{g}+\mathbf{u} C^{g}\right) \cdot \mathbf{n}=0 & \text { if } t>\left(t_{c}+t_{p}\right)\end{array}\right.$ 
For the initial condition, we assume that at the initial time $t:=0$ the FOUP and his atmosphere are not yet contamined i.e $C^{s}(\cdot, t=0)=0$ at $C^{g}(\cdot, t=0)=0$. The unknowns are the concentration $C^{s} \in \mathbb{R}^{2}$ in the polymer and the concentration $C^{g} \in \mathbb{R}^{2}$ in the FOUP's atmosphere. Pratically we assume that we study the model with a new FOUP for a first use. In the model, $\mathbf{D}_{g} \in \mathbb{R}_{+}$and $\mathbf{D}_{s} \in \mathbb{R}_{+}$are the gas diffusion coefficient in contaminant and solid media (polymer), which has no connection with the spatial location and no variation in time. $H(t-\varepsilon)$ denotes the Heaviside function, $C_{0}$ is the initial concentration in FOUP's atmosphere when the wafers post processed are finished to outgass the contaminant, $h_{0} \in \mathbb{R}_{+}$is the Henry constant and $\mathbf{n}$ is the outer unit normal vector on $\Gamma_{i}$, $T \in \mathbb{R}_{+}$is the final time and $\mathbf{q}_{1}$ and $\mathbf{q}_{2}$ are the source.

Because the concentration $C^{s} \in \mathbb{R}^{2}$ in the polymer depends of the concentration $C^{g} \in \mathbb{R}^{2}$ in the FOUP's atmosphere, we assume that the boundary condition that, on $\Gamma_{D}$ is a prescribed inflow concentration and at the interface, the two concentrations $C^{s} \in \mathbb{R}^{2}$ and $C^{g} \in \mathbb{R}^{2}$ will be equilibred by the Henry constant (Henry law). We will start by developing some analytical solution for the diffusion equation with uniform diffusivities in an unbounded domain and for very simple boundary conditions as developed by J.Cranck in [9]. Then we will solve the general form of the equation using the finite element method, a numerical technique of optimization, for any type of boundary condition. In these approaches, we interrelated the some assumptions. We suppose that, the FOUP and the membrane are homogenous and isotropic. The surface roughness of the internal surface of the FOUP or the membrane is neglected but we take into account this parameter when we use the Henry law at the interface. And then the diffusion coefficient is weakly in function of the concentration. We have, $\mathbf{D}_{s} \equiv \mathbf{D}_{s}\left(\nabla C^{s}\right)$ and $\mathbf{D}_{g} \equiv \mathbf{D}_{g}\left(\nabla C^{g}\right)$.

\subsection{Finite element methods}

We use the Galerkin finite element formulation of the problem given by equation (4). It is obtained by multiplying the equilibrium equation by an appropriate test function respectively $\phi$ and $\psi$ for the concentration $C^{s} \in \mathbb{R}^{2}$ and $C^{g} \in \mathbb{R}^{2}$ respectively, and by integrating in over the computational subdomain $\Omega_{s}$ and $\Omega_{g}$ respectively. We definite the following space:

$V^{s}:=\left\{C^{s} \in H^{1}\left(\Omega_{s}\right)^{2} ; \nabla C^{s}=0\right.$ on $\left.\Gamma_{s}\right\} ; \quad V^{g}:=\left\{C^{g} \in H^{1}\left(\Omega_{g}\right)^{2} ; \nabla C^{g}=0\right.$ on $\left.\Gamma_{g}\right\}$

In this case, we have

$$
\left\{\begin{aligned}
\int_{\Omega_{s}} \frac{\partial C^{s}}{\partial t} \phi d V^{\Omega_{i}} & =\int_{\Omega_{s}} \nabla \cdot\left(\mathbf{D}_{s} \nabla C^{s}\right) \phi d V^{\Omega_{i}}+\int_{\Omega_{s}} \mathbf{q}_{1} \phi d V^{\Omega_{i}} \\
\int_{\Omega_{g}} \frac{\partial C^{g}}{\partial t} \psi d V^{\Omega_{i}} & =\int_{\Omega_{g}} \nabla \cdot\left(\mathbf{D}_{g} \nabla C^{g}\right) \psi d V^{\Omega_{i}}-\int_{\Omega_{g}} \nabla \cdot\left(\mathbf{u} C^{g}\right) \psi d V^{\Omega_{i}}+\int_{\Omega_{s}} \mathbf{q}_{2} \psi d V^{\Omega_{i}}
\end{aligned}\right.
$$

Let us $H_{s}^{1}\left(\Omega_{s}\right)$ and $H_{s}^{1}\left(\Omega_{s}\right)$ be a functionnal space in which we are searching the solution in accordance with its regularity $H_{s}^{1}=\left\{\phi \in H^{1}\left(\Omega_{s}\right) \mid \phi=s \forall x \in \Gamma_{s}\right\}$ and $H_{s}^{1}=\left\{\psi \in H^{1}\left(\Omega_{g}\right) \mid \psi=s \forall x \in \Gamma_{g}\right\}$ where $H^{1}\left(\Omega_{s}\right)$ and $H^{1}\left(\Omega_{g}\right)$ are a Sobolev space, classicaly defined as $H^{1}\left(\Omega_{s}\right)=\left\{\phi \in L^{2}\left(\Omega_{s}\right),\|\nabla \phi\| \in L^{2}\left(\Omega_{s}\right)\right\}$ and $H^{1}\left(\Omega_{g}\right)=\left\{\phi \in L^{2}\left(\Omega_{g}\right),\|\nabla \phi\| \in\right.$ $\left.L^{2}\left(\Omega_{g}\right)\right\}$. Where $L^{2}\left(\Omega_{s}\right)$ and $L^{2}\left(\Omega_{g}\right)$ are the Hilbert vector space of the functions quadrically summable respectively on $\left(\Omega_{s}\right)$ and $\left(\Omega_{g}\right)$.

We have, $L^{2}\left(\Omega_{s}\right)=\left\{\left.\phi(x)\left|\int_{\Omega_{s}}\right| \phi(x)\right|^{2} d x<\infty\right\}$ and $L^{2}\left(\Omega_{g}\right)=\left\{\left.\psi(x)\left|\int_{\Omega_{g}}\right| \psi(x)\right|^{2} d x<\infty\right\}$.

The norm of these space are:

$\|\phi\|=\left(\int_{\Omega_{s}} \nabla \phi \cdot \nabla \phi d \mathbf{x}\right)^{\frac{1}{2}} ; \quad\|\psi\|=\left(\int_{\Omega_{g}} \nabla \psi \cdot \nabla \psi d \mathbf{x}\right)^{\frac{1}{2}}$

By using the Green's theorem, integration by parts of the equation (6) leads to,

$$
\left\{\begin{aligned}
\int_{\Omega_{s}} \frac{\partial C^{s}}{\partial t} \phi d V^{\Omega_{i}} & =-\int_{\Omega_{s}} \mathbf{D}_{s} \nabla C^{s} \cdot \nabla \phi d V^{\Omega_{i}}+\int_{\partial \Omega_{s}} \mathbf{D}_{s} \nabla C^{s} \cdot \mathbf{n} \phi d S^{\Omega_{i}}+\int_{\Omega_{s}} \mathbf{q} d V^{\Omega_{i}} \\
\int_{\Omega_{g}} \frac{\partial C^{g}}{\partial t} \psi d V^{\Omega_{i}} & =-\int_{\Omega_{g}} \mathbf{D}_{g} \nabla C^{g} \cdot \nabla \psi d V^{\Omega_{i}}+\int_{\partial \Omega_{g}} \mathbf{D}_{g} \nabla C^{g} \cdot \mathbf{n} \psi d S^{\Omega_{i}} \int_{\Omega_{g}} \nabla \cdot\left(\mathbf{u} C^{g}\right) \psi d V^{\Omega_{i}} \\
& +\int_{\Omega_{s}}^{\mathbf{q}_{2} \psi d V^{\Omega_{i}}}
\end{aligned}\right.
$$


By applying the Galerkin weighted residual methods and the Green's theorem, the variational formulation corresponding to the AMCs cross contamination is given by the equation (3). We now introduce the weak formulation of the AMCs model:

We find $C^{s} \in H^{1}\left(\Omega_{s}\right)$ and $C^{g} \in H^{1}\left(\Omega_{g}\right)$ such that,

$$
\left\{\begin{array}{l}
a_{1}\left(\frac{\partial C^{s}}{\partial t}, \phi\right)+b_{1}\left(C^{s}, \phi\right)=L_{1}(\phi) \quad \forall \phi \in H^{1}\left(\Omega_{s}\right) \\
a_{2}\left(\frac{\partial C^{g}}{\partial t}, \psi\right)+b_{2}\left(C^{g}, \psi\right)=L_{2}(\psi) \quad \forall \phi \in H^{1}\left(\Omega_{g}\right)
\end{array}\right.
$$

where

$$
\begin{aligned}
& L_{1}(\phi)=\int_{\Omega_{s}} \mathbf{q}_{1} \phi d V^{\Omega_{i}} ; \quad L_{2}(\psi)=\int_{\Omega_{s}} \mathbf{q}_{2} \psi d V^{\Omega_{i}}+\int_{\Omega_{g}} N_{0}^{g} \psi d S^{\Omega_{i}} ; \quad a_{1}\left(\frac{\partial C^{s}}{\partial t}, \phi\right)=\int_{\Omega_{s}} \frac{\partial C^{s}}{\partial t} \phi d V^{\Omega_{i}} \\
& a_{2}\left(\frac{\partial C^{g}}{\partial t}, \psi\right)=\int_{\Omega_{g}} \frac{\partial C^{g}}{\partial t} \psi d V^{\Omega_{i}} ; \quad b_{1}\left(C^{s}, \phi\right)=\int_{\Omega_{s}} \mathbf{D}_{s} \nabla C^{s} \cdot \nabla \phi d V^{\Omega_{i}}+\int_{\Gamma_{N}} h_{0} C^{g} \phi d S^{\Omega_{i}} \\
& b_{2}\left(C^{g}, \psi\right)=\int_{\Omega_{g}} \mathbf{D}_{g} \nabla C^{g} \cdot \nabla \psi d V^{\Omega_{i}}+\int_{\Omega_{g}} \nabla \cdot\left(\mathbf{u} C^{g}\right) \psi d V^{\Omega_{i}}+\int_{\Gamma_{N}} \frac{C^{s}}{h_{0}} \psi d S^{\Omega_{i}}+\int_{\Gamma_{D}} k_{c}\left[C_{0} H(t-\varepsilon)-C^{g}\right] \psi d S^{\Omega_{i}}
\end{aligned}
$$

The domain $\Omega_{s}$ and $\Omega_{g}$ are decomposed in finite number of subdomains $\Omega_{s}^{e}$ and $\Omega_{g}^{e}$ for each elements such that $\Omega_{s}:=\cup_{e} \Omega_{s}^{e}$ and $\Omega_{g}:=\cup_{e} \Omega_{g}^{e}$. Similarly, the boundary $\partial \Omega_{s}$ and $\partial \Omega_{g}$ are decomposed in $\partial \Omega_{s}^{e}$ and $\partial \Omega_{g}^{e}$. Finally the time interval is subdivised by $n$ subinterval.

For the spatial discretization, we assume the finite element partition $\mathcal{T}_{h}^{s}$ and $\mathcal{T}_{h}^{s}$ of $\Omega_{s}$ and $\Omega_{g}$ respectively into tetrahedral elements. Again for simplicity, we will consider that the finite element partition associated to $\mathcal{T}_{h}^{s}$ and $\mathcal{T}_{h}^{s}$ are uniform, $h$ is the size of the element domains. Let us $C_{h}^{s}$ and $C_{h}^{g}$ are the approximation solution of $C_{h}^{s}$ and $C_{h}^{g}$ respectively.

The Galerkin approximation (9) became:

Find $C_{h}^{s} \in H^{1 h}\left(\Omega_{g}\right)$ and $C_{h}^{g} \in H^{1 h}\left(\Omega_{g}\right)$ such that,

$$
\begin{cases}a_{1}\left(\frac{\partial C_{h}^{s}}{\partial t}, \phi_{h}\right)+b_{1}\left(C_{h}^{s}, \phi_{h}\right)=L_{1}\left(\phi_{h}\right) \quad \forall \phi_{h} \in H^{1 h}\left(\Omega_{s}\right) \\ a_{2}\left(\frac{\partial C_{h}^{g}}{\partial t}, \psi_{h}\right)+b_{2}\left(C^{g}, \psi_{h}\right)=L_{2}\left(\psi_{h}\right) \quad \forall \phi_{h} \in H^{1 h}\left(\Omega_{g}\right)\end{cases}
$$

Finally, we have a system of first order differential equation and using the matrix notation we have,

$$
\left[\begin{array}{cc}
\mathbb{M}^{s} & 0 \\
0 & \mathbb{M}^{g}
\end{array}\right]\left(\begin{array}{c}
\dot{\mathbf{C}}^{s} \\
\dot{\mathbf{C}}^{g}
\end{array}\right)+\left[\begin{array}{cc}
\mathbb{D}^{s} & h_{0} \\
h_{0}^{-1} & \mathbb{D}^{g}
\end{array}\right]\left(\begin{array}{l}
\mathbf{C}^{s} \\
\mathbf{C}^{g}
\end{array}\right)=\left(\begin{array}{c}
\mathbb{F}^{s} \\
\mathbb{F}^{g}
\end{array}\right)
$$

Where $\mathbf{C}^{s} \in \mathbb{R}^{2}$ and $\mathbf{C}^{g} \in \mathbb{R}^{2}$ are the unknowns concentration vectors on nodes, $\mathbb{M}^{s}$ and $\mathbb{M}^{g}$ are the time constant matrix, $\mathbb{F}^{s}$ and $\mathbb{F}^{g}$ are the source and external flux vector.

The system of ordinary differential equation (11) has to be integrated in time. Using the finite difference approximation and the explicite Euler scheme for $\dot{\mathbf{C}}^{s}$ and $\dot{\mathbf{C}}^{g}$,

We have,

$\Delta t=t_{n+1}-t_{n}$

$[0, T]=\bigcup_{i=1}^{n}\left[t_{i}, t_{i}+\Delta t\right]$

Where $T \in \mathbb{R}_{+}$is the range time and $\Delta t$ is the step time.

$\dot{\mathbf{C}}_{t+\Delta t}^{s}=\frac{\mathbf{C}_{t+\Delta t}^{s}-\mathbf{C}_{t}^{s}}{\Delta t} ; \quad \dot{\mathbf{C}}_{t+\Delta t}^{g}=\frac{\mathbf{C}_{t+\Delta t}^{g}-\mathbf{C}_{t}^{g}}{\Delta t} ;$ 
The system (11) can be written, at time $t+\Delta t$, as:

$$
\left[\begin{array}{cc}
\mathbb{M}^{s} & 0 \\
0 & \mathbb{M}^{g}
\end{array}\right]^{e}\left(\begin{array}{c}
\mathbf{C}_{n+1}^{s}-\mathbf{C}_{n}^{s} \\
\mathbf{C}_{n+1}^{g}-\mathbf{C}_{n}^{g}
\end{array}\right)^{e}+\Delta t\left[\begin{array}{cc}
\mathbb{D}^{s} & h_{0} \\
h_{0}^{-1} & \mathbb{D}^{g}
\end{array}\right]^{e}\left(\begin{array}{c}
\mathbf{C}_{n}^{s} \\
\mathbf{C}_{n}^{g}
\end{array}\right)^{e}=\Delta t\left(\begin{array}{c}
\mathbb{F}_{n}^{s} \\
\mathbb{F}_{n}^{g}
\end{array}\right)^{e}
$$

Where

$$
\begin{aligned}
& \mathbb{F}_{n}^{s}=\int_{\Omega_{s}^{e}} \mathbf{q N} d V^{\Omega_{i}} ; \quad \mathbb{F}_{n}^{g}=\int_{\Omega_{g}^{e}} N_{0}^{g} \mathbf{N} d V^{\Omega_{i}} ; \quad \mathbb{M}^{s}=\int_{\Omega_{s}^{e}} \mathbf{N}^{\mathbf{T}} \mathbf{N} d V^{\Omega_{i}} ; \quad \mathbb{M}^{g}=\int_{\Omega_{g}^{e}} \mathbf{N}^{\mathbf{T}} \mathbf{N} d V^{\Omega_{i}} \\
& \mathbb{D}^{s}=\int_{\Omega_{s}^{e}} \mathbf{D}_{s} \nabla \mathbf{N}^{\mathbf{T}} \cdot \nabla \mathbf{N} d V^{\Omega_{i}}+\int_{\Gamma_{N}} h_{0} \mathbf{N}^{\mathbf{T}} \mathbf{N} d S^{\Omega_{i}} ; \quad \mathbb{D}^{g}=\int_{\Omega_{g}^{e}} \mathbf{D}_{g} \nabla \mathbf{N}^{\mathbf{T}} \cdot \nabla \mathbf{N} d V^{\Omega_{i}}+\int_{\Omega_{g}^{e}} \nabla \cdot\left(\mathbf{u} \mathbf{N}^{\mathbf{T}}\right) \mathbf{N}^{\prime} d V^{\Omega_{i}} \\
& +\int_{\Gamma_{N}} \frac{\mathbf{N}^{\mathbf{T}}}{h_{0}} \mathbf{N} d S^{\Omega_{i}}+\int_{\Gamma_{D}} k_{c}\left[C_{0} H(t-\varepsilon)-\mathbf{N}^{\mathbf{T}}\right] \mathbf{N} d S^{\Omega_{i}}
\end{aligned}
$$

and $\mathbf{N}$ denote the linear interpolation function at each node.

And by using the assembling theory for all subdomain, we have:

$\bigcup_{e=1}^{m}\left[\begin{array}{cc}\tilde{\mathbb{M}}^{s} & 0 \\ 0 & \tilde{\mathbb{M}}^{g}\end{array}\right]^{e}\left(\begin{array}{c}\mathbf{C}_{n+1}^{s}-\mathbf{C}_{n}^{s} \\ \mathbf{C}_{n+1}^{g}-\mathbf{C}_{n}^{g}\end{array}\right)^{e}+\bigcup_{e=1}^{m} \Delta t\left[\begin{array}{cc}\tilde{\mathbb{D}}^{s} & h_{0} \\ h_{0}^{-1} & \tilde{\mathbb{D}}^{g}\end{array}\right]^{e}\left(\begin{array}{c}\mathbf{C}_{n}^{s} \\ \mathbf{C}_{n}^{g}\end{array}\right)^{e}=\bigcup_{e=1}^{m} \Delta t\left(\begin{array}{c}\tilde{\mathbb{F}}_{n}^{s} \\ \tilde{\mathbb{F}}_{n}^{g}\end{array}\right)^{e}$

Where $\tilde{\mathbb{M}}_{i j}^{s, g}=\sum_{i, j}^{p_{\text {nodes }}} \mathbb{M}_{i j}^{s, g}, \tilde{\mathbb{D}}_{i j}^{s, g}=\sum_{i, j}^{p_{\text {nodes }}} \mathbb{D}_{i j}^{s, g}, \tilde{\mathbb{F}}_{j}^{s, g}=\sum_{j}^{p_{\text {nodes }}} \mathbb{F}_{j}^{s, g}$,

By assumming this switch condition, the matrix components in equation (15), can be expressed as follows:

$$
\begin{aligned}
\mathbb{F}_{n}^{s} & =\int_{\Omega_{s}^{e}} \mathbf{q} \mathbf{N} d V^{\Omega_{i}} ; \quad \mathbb{F}_{n}^{g}=0 ; \quad \mathbb{M}^{s}=\int_{\Omega_{s}^{e}} \mathbf{N}^{\mathbf{T}} \mathbf{N} d V^{\Omega_{i}} ; \quad \mathbb{M}^{g}=\int_{\Omega_{g}^{e}} \mathbf{N}^{\mathbf{T}} \mathbf{N} d V^{\Omega_{i}} \\
\mathbb{D}^{s} & =\int_{\Omega_{s}^{e}} \mathbf{D}_{s} \nabla \mathbf{N}^{\mathbf{T}} \cdot \nabla \mathbf{N} d V^{\Omega_{i}}+\int_{\Gamma_{N}} h_{0} \mathbf{N}^{\mathbf{T}} \mathbf{N} d S^{\Omega_{i}} \\
\mathbb{D}^{g} & =\int_{\Omega_{g}^{e}} \mathbf{D}_{g} \nabla \mathbf{N}^{\mathbf{T}} \cdot \nabla \mathbf{N} d V^{\Omega_{i}}+\int_{\Omega_{g}^{e}} \nabla \cdot\left(\mathbf{u} \mathbf{N}^{\mathbf{T}}\right) \mathbf{N} d V^{\Omega_{i}}+\int_{\Gamma_{N}} \frac{\mathbf{N}^{\mathbf{T}}}{h_{0}} \mathbf{N} d S^{\Omega_{i}}
\end{aligned}
$$

\section{Model with temperature effect}

\subsection{Introduction}

In this section we will study the temperature effect on the AMCs cross-contamination between wafer and FOUP. For the first approximation, we use the Arrhenius law, this law assume that just the diffusion coefficient change with the temperature. The diffusion coefficient is in function of the temperature. The mathematical model including this law is traduced by the equation (17). The Arrhenius law applied in the polymer and the contaminant is given as:

$\mathbf{D}_{s, g}^{*}\left(T^{*}\right)=\mathbf{D}_{0 s, 0 g}^{*} \exp \left(\frac{E}{R T}-\frac{E}{R T^{*}}\right)$

Where $\mathbf{D}_{s, g}^{*}$ and $\mathbf{D}_{0 s, 0 g}^{*}$ denote respectively the diffusion coefficient in the polymer/contaminant at the temperature $T^{*}$ and the reference diffusion coefficient in the polymer/contaminant at the reference temperature $T$. 


\subsection{Mathematical model using Arrhenius law}

We assume that the advection parts and reaction time scale are slow compared to the diffusive time scale. The model for transient contaminant transfer between the wafer and the internal part of the FOUP is given by:

$$
\left\{\begin{array}{llr}
\frac{\partial C^{s *}}{\partial t} & =\nabla \cdot\left(\mathbf{D}_{s}^{*}\left(T^{*}\right) \nabla C^{s *}\right)+\mathbf{q}_{1}^{*} & \text { in }\left(\Omega_{s} \times[0, T]\right) \\
C^{s *} & =h_{0}^{*} C^{* g} & \text { on }\left(\Gamma_{N}\right) \\
\mathbf{D}_{s}^{*}\left(T^{*}\right) \nabla C^{s *} \cdot \mathbf{n} & =0 & \text { on }\left(\partial \Omega_{s}-\Gamma_{N}\right) \\
\mathbf{D}_{s}^{*}\left(T^{*}\right) & =\mathbf{D}_{0 s}^{*} \exp \left(\frac{E}{R T}-\frac{E}{R T^{*}}\right) & \text { in }\left(\Omega_{s} \times[0, T]\right) \\
\frac{\partial C^{g *}}{\partial t} & =\nabla \cdot\left(\mathbf{D}_{g}^{*}\left(T^{*}\right) \nabla C^{g *}\right)-\nabla \cdot\left(\mathbf{u}^{*} C^{g *}\right)+\mathbf{q}_{2}^{*} & \text { in }\left(\Omega_{g} \times[0, T]\right) \\
\left(-\mathbf{D}_{g}^{*}\left(T^{*}\right) \nabla C^{g *}+\mathbf{u}^{*} C^{g *}\right) \cdot \mathbf{n} & =N_{0}^{g}+k_{c}\left[C_{0} H(t-\varepsilon)-C^{g *}\right] & \text { on }\left(\Gamma_{D}\right) \\
C^{g *} & =\frac{C^{s *}}{h_{0}^{*}} & \text { on }\left(\Gamma_{N}\right) \\
\left(-\mathbf{D}_{g}^{*}\left(T^{*}\right) \nabla C^{g}+\mathbf{u}^{*} C^{g *}\right) \cdot \mathbf{n} & =0 & \text { on } \partial \bar{\Omega}_{g} \\
\mathbf{D}_{g}^{*}\left(T^{*}\right) & =\mathbf{D}_{0 g}^{*} \exp \left(\frac{E}{R T}-\frac{E}{R T^{*}}\right) & \text { in }\left(\Omega_{s} \times[0, T]\right)
\end{array}\right.
$$

Where $\partial \bar{\Omega}_{g}=\left(\partial \Omega_{g}-\left(\Gamma_{N} \cup \Gamma_{D}\right)\right)$

\section{3. $\quad$ Finite element methods}

We use the Galerkin finite element formulation for numerical solution of the problem. Classically, it is obtained by multiplying the equilibrium equation by an appropriate test function respectively $\phi^{*}$ and $\psi^{*}$ for the concentration $C^{s *} \in \mathbb{R}^{2}$ and $C^{g *} \in \mathbb{R}^{2}$ respectively, and by integrating respectively over the computational subdomain $\Omega_{s *}$ and $\Omega_{g *}$.

We definite the following space:

$V^{s *}:=\left\{C^{s} \in H^{1}\left(\Omega_{s *}\right)^{2} ; \nabla C^{s *}=0\right.$ on $\left.\Gamma_{s *}\right\} ; \quad V^{g *}:=\left\{C^{g} \in H^{1}\left(\Omega_{g *}\right)^{2} ; \nabla C^{g *}=0\right.$ on $\left.\Gamma_{g *}\right\}$

In this case, we have:

$$
\begin{cases}\int_{\Omega_{s}} \frac{\partial C^{s *}}{\partial t} \phi^{*} d V^{\Omega_{i}} & =\int_{\Omega_{s}} \nabla \cdot\left(\mathbf{D}_{s}^{*}\left(T^{*}\right) \nabla C^{s *}\right) \phi^{*} d V^{\Omega_{i}}+\int_{\Omega_{s}} \mathbf{q}_{1}^{*} \phi^{*} d V^{\Omega_{i}} \\ \int_{\Omega_{g}} \frac{\partial C^{g *}}{\partial t} \psi^{*} d V^{\Omega_{i}} & =\int_{\Omega_{g}} \nabla \cdot\left(\mathbf{D}_{g}^{*}\left(T^{*}\right) \nabla C^{g *}\right) \psi^{*} d V^{\Omega_{i}}-\int_{\Omega_{g}} \nabla \cdot\left(\mathbf{u}^{*} C^{g *}\right) \psi^{*} d V^{\Omega_{i}}+\int_{\Omega_{s}} \mathbf{q}_{2}^{*} \psi^{*} d V^{\Omega_{i}} \\ \mathbf{D}_{s, g}^{*}\left(T^{*}\right) & =\mathbf{D}_{0 s, 0 g}^{*} \exp \left(\frac{E}{R T}-\frac{E}{R T^{*}}\right)\end{cases}
$$

Let us $H_{s}^{1}\left(\Omega_{s}\right)$ and $H_{s}^{1}\left(\Omega_{s}\right)$ be a functional space in which we are searching the solution in accordance with its regularity $H_{s}^{1}=\left\{\phi^{*} \in H^{1}\left(\Omega_{s}\right) \mid \phi^{*}=s \forall x \in \Gamma_{s}\right\}$ and $H_{s}^{1}=\left\{\psi^{*} \in H^{1}\left(\Omega_{g}\right) \mid \psi^{*}=s \forall x \in \Gamma_{g}\right\}$ where $H^{1}\left(\Omega_{s}\right)$ and $H^{1}\left(\Omega_{g}\right)$ are a Sobolev space, classicaly defined as $H^{1}\left(\Omega_{s}\right)=\left\{\phi^{*} \in L^{2}\left(\Omega_{s}\right),\left\|\nabla \phi^{*}\right\| \in L^{2}\left(\Omega_{s}\right)\right\}$ and $H^{1}\left(\Omega_{g}\right)=\left\{\phi^{*} \in\right.$ $\left.L^{2}\left(\Omega_{g}\right),\left\|\nabla \phi^{*}\right\| \in L^{2}\left(\Omega_{g}\right)\right\}$. Where $L^{2}\left(\Omega_{s}\right)$ and $L^{2}\left(\Omega_{g}\right)$ are the Hilbert vector space of the functions quadrically summable respectively on $\left(\Omega_{s}\right)$ and $\left(\Omega_{g}\right)$.

We have, $L^{2}\left(\Omega_{s}\right)=\left\{\left.\phi^{*}(x)\left|\int_{\Omega_{s}}\right| \phi^{*}(x)\right|^{2} d x<\infty\right\}$ and $L^{2}\left(\Omega_{g}\right)=\left\{\left.\psi^{*}(x)\left|\int_{\Omega_{g}}\right| \psi^{*}(x)\right|^{2} d x<\infty\right\}$.

The norm of these space are:

$\left\|\phi^{*}\right\|=\left(\int_{\Omega_{s}} \nabla \phi^{*} \cdot \nabla \phi^{*} d \mathbf{x}\right)^{\frac{1}{2}} ; \quad\left\|\psi^{*}\right\|=\left(\int_{\Omega_{g}} \nabla \psi^{*} \cdot \nabla \psi^{*} d \mathbf{x}\right)^{\frac{1}{2}}$

By using the Green's theorem, integration by parts leads to,

$$
\left\{\begin{aligned}
\int_{\Omega_{s}} \frac{\partial C^{s *}}{\partial t} \phi^{*} d V^{\Omega_{i}} & =-\int_{\Omega_{s}} \mathbf{D}_{s}^{*}\left(T^{*}\right) \nabla C^{s *} \cdot \nabla \phi^{*} d V^{\Omega_{i}}+\int_{\partial \Omega_{s}} \mathbf{D}_{s}^{*}\left(T^{*}\right) \nabla C^{s} \cdot \mathbf{n} \phi^{*} d S^{\Omega_{i}}+\int_{\Omega_{s}} \mathbf{q}_{1}^{*} \phi^{*} d V^{\Omega_{i}} \\
\int_{\Omega_{g}} \frac{\partial C^{g *}}{\partial t} \psi^{*} d V^{\Omega_{i}} & =-\int_{\Omega_{g}} \mathbf{D}_{g}^{*}\left(T^{*}\right) \nabla C^{g *} \cdot \nabla \psi^{*} d V^{\Omega_{i}}+\int_{\partial \Omega_{g}} \mathbf{D}_{g}^{*}\left(T^{*}\right) \nabla C^{g *} \cdot \mathbf{n} \psi^{*} d S^{\Omega_{i}} \\
& -\int_{\Omega_{g}} \nabla \cdot\left(\mathbf{u}^{*} C^{g *}\right) \psi^{*} d V^{\Omega_{i}}+\int_{\Omega_{s}} \mathbf{q}_{2}^{*} \psi^{*} d V^{\Omega_{i}} \\
\mathbf{D}_{s, g}^{*}\left(T^{*}\right) & =\mathbf{D}_{0 s, 0 g}^{*} \exp \left(\frac{E}{R T}-\frac{E}{R T^{*}}\right)
\end{aligned}\right.
$$


By applying the Galerkin weighted residual methods and the Green's theorem , the variational formulation corresponding the AMCs cross contamination is given by the equation (1). We now introduce the weak formulation of the AMCs model:

We find $C^{s *} \in H^{1}\left(\Omega_{s}\right)$ and $C^{g *} \in H^{1}\left(\Omega_{g}\right)$ such that,

$$
\left\{\begin{array}{rlrl}
a_{1}^{*}\left(\frac{\partial C^{s *}}{\partial t}, \phi^{*}\right)+b_{1}^{*}\left(C^{s *}, \phi^{*}\right) & =L_{1}^{*}\left(\phi^{*}\right) & & \forall \phi^{*} \in H^{1}\left(\Omega_{s}\right) \\
a_{2}^{*}\left(\frac{\partial C^{g *}}{\partial t}, \psi^{*}\right)+b_{2}^{*}\left(C^{g *}, \psi^{*}\right) & =L_{2}^{*}\left(\psi^{*}\right) & \forall \phi^{*} \in H^{1}\left(\Omega_{g}\right) \\
\mathbf{D}_{s, g}^{*}\left(T^{*}\right) & =\mathbf{D}_{0 s, 0 g}^{*} \exp \left(\frac{E}{R T}-\frac{E}{R T^{*}}\right) &
\end{array}\right.
$$

where

$$
\begin{aligned}
& L_{1}^{*}\left(\phi^{*}\right)=\int_{\Omega_{s}} \mathbf{q}_{1}^{*} \phi^{*} d V^{\Omega_{i}} ; \quad L_{2}^{*}\left(\psi^{*}\right)=\int_{\Omega_{s}} \mathbf{q}_{2}^{*} \psi^{*} d V^{\Omega_{i}}+\int_{\Omega_{g}} N_{0}^{g} \psi^{*} d S^{\Omega_{i}} ; \quad a_{1}^{*}\left(\frac{\partial C^{s *}}{\partial t}, \phi^{*}\right)=\int_{\Omega_{s}} \frac{\partial C^{s *}}{\partial t} \phi^{*} d V^{\Omega_{i}} \\
& a_{2}^{*}\left(\frac{\partial C^{g *}}{\partial t}, \psi^{*}\right)=\int_{\Omega_{g}} \frac{\partial C^{g}}{\partial t} \psi^{*} d V^{\Omega_{i}} ; \quad b_{1}^{*}\left(C^{s *}, \phi^{*}\right)=\int_{\Omega_{s}} \mathbf{D}_{s *}\left(T^{*}\right) \nabla C^{s *} \cdot \nabla \phi^{*} d V^{\Omega_{i}}+\int_{\Gamma_{N}} h_{0}^{*} C^{g *} \phi^{*} d S^{\Omega_{i}} \\
& b_{2}^{*}\left(C^{g *}, \psi^{*}\right)=\int_{\Omega_{g}} \mathbf{D}_{g}^{*}\left(T^{*}\right) \nabla C^{g *} \cdot \nabla \psi^{*} d V^{\Omega_{i}}+\int_{\Omega_{g}} \nabla \cdot\left(\mathbf{u}^{*} C^{g *}\right) \psi^{*} d V^{\Omega_{i}} \\
& +\int_{\Gamma_{N}} \frac{C^{s *}}{h_{0}^{*}} \psi^{*} d S^{\Omega_{i}}+\int_{\Gamma_{D}} k_{c}\left[C_{0} H(t-\varepsilon)-C^{g *}\right] \psi^{*} d S^{\Omega_{i}}
\end{aligned}
$$

The domain $\Omega_{s}$ and $\Omega_{g}$ are decomposed in finite number of subdomains $\Omega_{s}^{e}$ and $\Omega_{g}^{e}$ for each elements. Similarly, the boundary $\partial \Omega_{s}$ and $\partial \Omega_{g}$ are decomposed in $\partial \Omega_{s}^{e}$ and $\partial \Omega_{g}^{e}$. Finally the time interval is subdivised by $n$ subinterval.

For the spatial discretization, we assume the finite element partition $\mathcal{T}_{h}^{s}$ and $\mathcal{T}_{h}^{s}$ of $\Omega_{s}$ and $\Omega_{g}$ respectively into tetrahedral elements. Again for simplicity, we will assume that the finite element partition associated to $\mathcal{T}_{h}^{s}$ and $\mathcal{T}_{h}^{s}$ are uniform, $h$ is the size of the element domains. Let us $C_{h}^{s *}$ and $C_{h}^{g *}$ be the approximations solutions of $C_{h}^{s *}$ and $C_{h}^{g *}$ respectively.

The Galerkin approximation above became:

Find $C_{h}^{s *} \in H^{1 h}\left(\Omega_{g}\right)$ and $C_{h}^{g *} \in H^{1 h}\left(\Omega_{g}\right)$ such that,

$$
\left\{\begin{array}{rlrl}
a_{1}^{*}\left(\frac{\partial C_{h}^{s *}}{\partial t}, \phi_{h}^{*}\right)+b_{1}^{*}\left(C_{h}^{s *}, \phi_{h}^{*}\right) & =L_{1}^{*}\left(\phi_{h}^{*}\right) & & \forall \phi_{h}^{*} \in H^{1 h}\left(\Omega_{s}\right) \\
a_{2}^{*}\left(\frac{\partial C_{h}^{g *}}{\partial t}, \psi_{h}^{*}\right)+b_{2}^{*}\left(C^{g *}, \psi_{h}^{*}\right) & =L_{2}^{*}\left(\psi_{h}^{*}\right) & & \forall \phi_{h}^{*} \in H^{1 h}\left(\Omega_{g}\right) \\
\mathbf{D}_{s, g}^{*}\left(T^{*}\right) & =\mathbf{D}_{0 s, 0 g}^{*} \exp \left(\frac{E}{R T}-\frac{E}{R T^{*}}\right) &
\end{array}\right.
$$

Finally, we have a system of first order differential equation and using the matrix notation we have,

$\left[\begin{array}{cc}\mathbb{M}^{s *} & 0 \\ 0 & \mathbb{M}^{g *}\end{array}\right]\left(\begin{array}{c}\dot{\mathbf{C}}^{s *} \\ \dot{\mathbf{C}}^{g *}\end{array}\right)+\left[\begin{array}{cc}\mathbb{D}^{s *} & h_{0}^{*} \\ h_{0}^{-1 *} & \mathbb{D}^{g *}\end{array}\right]\left(\begin{array}{c}\mathbf{C}^{s *} \\ \mathbf{C}^{g *}\end{array}\right)=\left(\begin{array}{c}\mathbb{F}^{s *} \\ \mathbb{F}^{g *}\end{array}\right)$

Where $\mathbf{C}^{s *} \in \mathbb{R}^{2}$ and $\mathbf{C}^{g *} \in \mathbb{R}^{2}$ are the unknowns concentration vectors on nodes, $\mathbb{M}^{s *}$ and $\mathbb{M}^{g}$ are the time constant matrix, $\mathbb{F}^{s *}$ and $\mathbb{F}^{g *}$ are the source and external flux vectors.

Using the same methods as defined in the equation (12), (13) and (14) in the section 3.4, the system can be written at time $t+\Delta t$ as:

$\left[\begin{array}{cc}\mathbb{M}^{s *} & 0 \\ 0 & \mathbb{M}^{g *}\end{array}\right]^{e}\left(\begin{array}{c}\mathbf{C}_{n+1}^{s *}-\mathbf{C}_{n}^{s *} \\ \mathbf{C}_{n+1}^{g *}-\mathbf{C}_{n}^{g *}\end{array}\right)^{e}+\Delta t\left[\begin{array}{cc}\mathbb{D}^{s *} & h_{0}^{*} \\ h_{0}^{-1 *} & \mathbb{D}^{g *}\end{array}\right]^{e}\left(\begin{array}{c}\mathbf{C}_{n}^{s *} \\ \mathbf{C}_{n}^{g *}\end{array}\right)^{e}=\Delta t\left(\begin{array}{c}\mathbb{F}_{n}^{s *} \\ \mathbb{F}_{n}^{g *}\end{array}\right)^{e}$

Where

$$
\begin{aligned}
& \mathbb{F}_{n}^{s *}=\int_{\Omega_{s}^{e}} \mathbf{q}_{1}^{*} \mathbf{N} d V^{\Omega_{i}} ; \quad \mathbb{F}_{n}^{g *}=\int_{\Omega_{s}^{e}} \mathbf{q}_{1}^{*} \mathbf{N} d V^{\Omega_{i}}+\int_{\Omega_{g}^{e}} N_{0}^{g} \mathbf{N} d V^{\Omega_{i}} ; \quad \mathbb{M}^{s *}=\int_{\Omega_{s}^{e}} \mathbf{N}^{\mathbf{T}} \mathbf{N} d V^{\Omega_{i}} ; \\
& \mathbb{M}^{g *}=\int_{\Omega_{g}^{e}} \mathbf{N}^{\mathbf{T}} \mathbf{N} d V^{\Omega_{i}} ; \quad \mathbb{D}^{s *}=\int_{\Omega_{s}^{e}} \mathbf{D}_{s}^{*}\left(T^{*}\right) \nabla \mathbf{N}^{\mathbf{T}} \cdot \nabla \mathbf{N} d V^{\Omega_{i}}+\int_{\Gamma_{N}} h_{0}^{*} \mathbf{N}^{\mathbf{T}} \mathbf{N} d S^{\Omega_{i}} \\
& \mathbb{D}^{g *}=\int_{\Omega_{g}^{e}} \mathbf{D}_{g}^{*}\left(T^{*}\right) \nabla \mathbf{N}^{\mathbf{T}} \cdot \nabla \mathbf{N} d V^{\Omega_{i}}+\int_{\Omega_{g}^{e}} \nabla \cdot\left(\mathbf{u}^{*} \mathbf{N}^{\mathbf{T}}\right) \mathbf{N} d V^{\Omega_{i}}+\int_{\Gamma_{N}} \frac{\mathbf{N}^{\mathbf{T}}}{h_{0}^{*}} \mathbf{N} d S^{\Omega_{i}}+\int_{\Gamma_{D}} k_{c}\left[C_{0} H(t-\varepsilon)-\mathbf{N}^{\mathbf{T}}\right] \mathbf{N} d S^{\Omega_{i}}
\end{aligned}
$$


and $\mathbf{N}$ denote the linear interpolation function at each node.

And by using the assembly theory for all subdomain, we have:

$\bigcup_{e=1}^{m}\left[\begin{array}{cc}\tilde{\mathbb{M}}^{s *} & 0 \\ 0 & \tilde{\mathbb{M}}^{g *}\end{array}\right]^{e}\left(\begin{array}{c}\mathbf{C}_{n+1}^{s *}-\mathbf{C}_{n}^{s *} \\ \mathbf{C}_{n+1}^{g *}-\mathbf{C}_{n}^{g *}\end{array}\right)^{e}+\bigcup_{e=1}^{m} \Delta t\left[\begin{array}{cc}\tilde{\mathbb{D}}^{s *} & h_{0}^{*} \\ h_{0}^{-1 *} & \tilde{\mathbb{D}^{g *}}\end{array}\right]^{e}\left(\begin{array}{c}\mathbf{C}_{n}^{s *} \\ \mathbf{C}_{n}^{g *}\end{array}\right)^{e}=\bigcup_{e=1}^{m} \Delta t\left(\begin{array}{c}\tilde{\mathbb{F}}_{n}^{s *} \\ \tilde{\mathbb{F}}_{n}^{g *}\end{array}\right)^{e}$

Where $\tilde{\mathbb{M}}_{i j}^{s *, g *}=\sum_{i, j}^{p_{\text {nodes }}} \mathbb{M}_{i j}^{s *, g *}, \tilde{\mathbb{D}}_{i j}^{s *, g *}=\sum_{i, j}^{p_{\text {nodes }}} \mathbb{D}_{i j}^{s *, g *}, \tilde{\mathbb{F}}_{j}^{s *, g *}=\sum_{j}^{p_{\text {nodes }}} \mathbb{F}_{j}^{s *, g *}$,

We use the same conditions as defined in the equation (23) and (24). This method is defined to simulate the contaminant concentration $C_{0} \in \mathbb{R}_{+}$during the contamination time $t_{c} \in \mathbb{R}_{+}$, and when the contamination time is finished the wafer surface is considered as a neutral surface. In fact, we use the same conditions as defined in the section (3.3). It consist to write the condition as a flux inflow and we use the parameter $k_{c} \in \mathbb{R}_{+}$to switch the two conditions. In this case, the boundary condition in wafer surface is given by:

$\left(-\mathbf{D}_{g}^{*}\left(T^{*}\right) \nabla C^{g *}+\mathbf{u}^{*} C^{g *}\right) \cdot \mathbf{n}=N_{0}^{g}+k_{c}\left[C_{0} H(t-\varepsilon)-C^{g *}\right]$

We assume that $N_{0}^{g}=0$ (no initial flux) and we dynamic boundary condition with a laminar gas flow on this boundary $(\mathbf{u}=0)$. When $k_{c} \in \mathbb{R}_{+}$is sufficiently large, we have the Dirichlet condition i.e $C_{0} H(t-\varepsilon) \simeq C^{g *}$ and if $k_{c}=0$ we have the Neumann's boundary condition (for the neutral surface of the wafer), i.e $\left(-\mathbf{D}_{g}^{*}\left(T^{*}\right) \nabla C^{g *}+\right.$ $\left.\mathbf{u}^{*} C^{g *}\right) \cdot \mathbf{n}=0$. So we need to conditionate $C_{0} \in \mathbb{R}_{+}$and $k_{c} \in \mathbb{R}_{+}$a parametric study was made to ensure the stability.

We have,

if $\left\{\begin{array}{lrl}k_{c} \gg 1 \Rightarrow C^{g *} \simeq C_{0} H(t-\varepsilon) & \text { if } 0<t \leq\left(t_{c}+t_{p}\right) \\ k_{c}=0 \Rightarrow\left(-\mathbf{D}_{g}^{*}\left(T^{*}\right) \nabla C^{g *}+\mathbf{u}^{*} C^{g *}\right) \cdot \mathbf{n}=0 & \text { if } t>\left(t_{c}+t_{p}\right)\end{array}\right.$

By assumming this switch conditions, the matrix components in equation (21), expressed as:

$$
\begin{aligned}
& \mathbb{F}_{n}^{s *}=\int_{\Omega_{s}^{e}} \mathbf{q}_{1}^{*} \mathbf{N} d V^{\Omega_{i}} ; \quad \mathbb{F}_{n}^{g *}=\int_{\Omega_{s}^{e}} \mathbf{q}_{2}^{*} \mathbf{N} d V^{\Omega_{i}} ; \quad \mathbb{M}^{M^{*}}=\int_{\Omega_{s}^{e}} \mathbf{N}^{\mathbf{T}} \mathbf{N} d V^{\Omega_{i}} ; \quad \mathbb{M}^{g *}=\int_{\Omega_{g}^{e}} \mathbf{N}^{\mathbf{T}} \mathbf{N} d V^{\Omega_{i}} \\
& \mathbb{D}^{s *}=\int_{\Omega^{e}} \mathbf{D}_{s}^{*}\left(T^{*}\right) \nabla \mathbf{N}^{\mathbf{T}} \cdot \nabla \mathbf{N} d V^{\Omega_{i}}+\int_{\Gamma_{N}} h_{0}^{*} \mathbf{N}^{\mathbf{T}} \mathbf{N} d S^{\Omega_{i}} \\
& \mathbb{D}^{g *}=\int_{\Omega_{g}^{e}} \mathbf{D}_{g}^{*}\left(T^{*}\right) \nabla \mathbf{N}^{\mathbf{T}} \cdot \nabla \mathbf{N} d V^{\Omega_{i}}+\int_{\Omega_{s *}^{e}} \nabla \cdot\left(\mathbf{u}^{*} \mathbf{N}^{\mathbf{T}}\right) \mathbf{N} d V^{\Omega_{i}}+\int_{\Gamma_{N}} \frac{\mathbf{N}^{\mathbf{T}}}{h_{0}^{*}} \mathbf{N} d S^{\Omega_{i}}
\end{aligned}
$$

\section{Mathematical model with heat effects}

\subsection{Introduction}

In this section we will study the temperature effect on the AMCs cross-contamination by using the heat equation. The diffusion coefficient is in function of the temperature.

The Fourier's law is given by the equation (29).

$J_{T}^{i}=-\kappa_{s} \nabla T^{i}$

And the conservation equation is given by:

$\rho^{s} c^{s} \frac{\partial T^{i}}{\partial t}=-\nabla \cdot J_{T}^{i}-\nabla \cdot \mathbf{u} T^{i}+\rho^{s} \mathbf{r}$

The mathematical model including this law is traduced by the equation. We introduce in the model already defined at the section (3), the heat conduction and at the FOUP (polymer), at the FOUP's atmosphere we assume that the temperature is constant. According to the geometry simplification and the notations already defined at the section (2), the model using the temperature effect is given by the system (40) with the REV the same in figure (2). 


\subsection{Model using the heat equation}

The mathematical model of the AMCs cross contamination with the temperature effect can be expressed as:

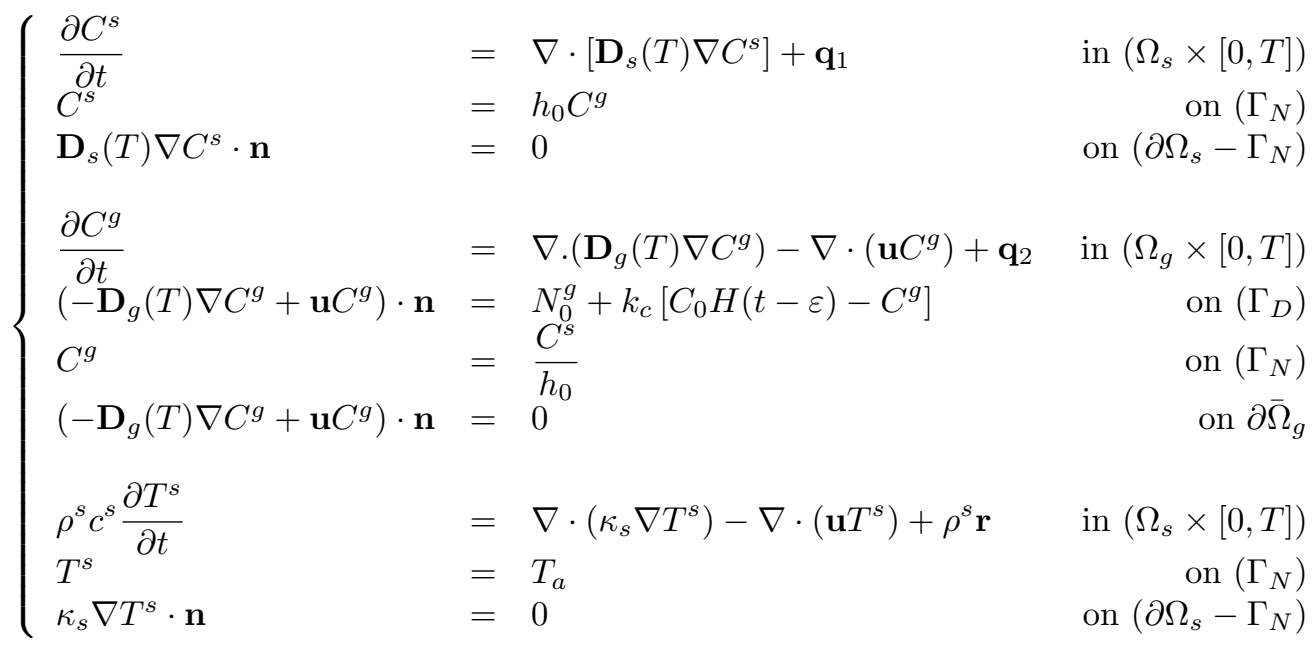

where $\partial \bar{\Omega}_{g}=\left(\partial \Omega_{g}-\left(\Gamma_{N} \cup \Gamma_{D}\right)\right)$.

The initial conditions are defined as: the initial time $t:=0$ the FOUP and his atmspehere are not yet contaminned i.e $C^{s}(\cdot, t=0)=0$ at $C^{s}(\cdot, t=0)=0$. At the initial time, we assume that the initial temperatures are the ambiant temperatures (absolute temperature) $T^{\infty}$ i.e $T^{s}(\cdot, t=0)=T^{\infty}$ at $T^{g}(\cdot, t=0)=T^{\infty}$. Pratically, we assume that we study with a new FOUP for a first use. In the model as defined above, $\kappa_{s} \in \mathbb{R}_{+}$denote the conduction coefficient, $T^{s} \in \mathbb{R}_{+}$the temperature at the FOUPs. We assume that the diffusion coefficient is in function of the temperature $\mathbf{D}_{s}=\mathbf{D}_{s}(T)$ and $T^{a} \in \mathbb{R}_{+}$denotes the temperature of cleaner fluid prescribed at the internal surface of the FOUP. We use the same boundary condition in the model. This concentration conditions will be defined on $\Gamma_{D}$ and we use the same boundary condition on the other boundary. And for the temperature condition in $\Gamma_{N}$ we have a prescribed temperature (the temperature of the cleaning fluid) $T_{a} \in \mathbb{R}_{+}$. The industrial conditions is characterized by many step. For each steps the difference is the time characteristic and the boundary condition in $\Gamma_{D}$.

\subsection{Finite element methods}

We use the Galerkin finite element formulation for numerical solution of the problem given by equation (30). It is obtained by multiplying the equilibrium equation by an appropriate test function respectively $\phi, \psi$ and $\chi$ for the concentration $C^{s} \in \mathbb{R}^{2}, C^{g} \in \mathbb{R}^{2}$ and the temperature $T^{s}$ respectively, and by integrating over the computational domain. We definite the following space:

$$
\begin{aligned}
V^{s}:=\left\{C^{s} \in H^{1}\left(\Omega_{s}\right)^{2} ; \nabla C^{s}=0 \text { on } \Gamma_{s}\right\} ; \quad V^{g}:=\left\{C^{g} \in H^{1}\left(\Omega_{g}\right)^{2} ; \nabla C^{g}=0 \text { on } \Gamma_{g}\right\} ; \\
V^{T^{s}}:=\left\{T^{s} \in H^{1}\left(\Omega_{s}\right)^{2} ; \nabla T^{s}=0 \text { on } \Gamma_{s}\right\}
\end{aligned}
$$

We use the classical finite element formulation for the numerical solution of the problem given by equation (40) ca be written:

$$
\begin{cases}\int_{\Omega_{s}} \frac{\partial C^{s}}{\partial t} \phi^{k} d V^{\Omega_{i}} & =\int_{\Omega_{s}} \nabla \cdot\left(\mathbf{D}_{s}(T) \nabla C^{s}\right) \phi^{k} d V^{\Omega_{i}}+\int_{\Omega_{s}} \mathbf{q}_{1} \phi^{k} d V^{\Omega_{i}} \\ \int_{\Omega_{g}} \frac{\partial C^{g}}{\partial t} \psi^{k} d V^{\Omega_{i}} & =\int_{\Omega_{g}} \nabla \cdot\left(\mathbf{D}_{g} \nabla C^{g}\right) \psi^{k} d V^{\Omega_{i}}-\int_{\Omega_{g}} \nabla \cdot\left(\mathbf{u} C^{g}\right) \psi^{k} d V^{\Omega_{i}} \int_{\Omega_{g}} \mathbf{q}_{2} \psi^{k} d V^{\Omega_{i}} \\ \int_{\Omega_{s}} \rho^{s} c^{s} \frac{\partial T^{s}}{\partial t} \chi^{k} d V^{\Omega_{i}} & =\int_{\Omega_{s}}^{\nabla} \nabla \cdot\left(\kappa_{s} \nabla T^{s}\right) \chi^{k} d V^{\Omega_{i}}-\int_{\Omega_{g}} \nabla \cdot\left(\mathbf{u} T^{s}\right) \chi^{k} d V^{\Omega_{i}} \int_{\Omega_{s}} \rho^{s} \mathbf{r} \chi^{k} d V^{\Omega_{i}}\end{cases}
$$

Let us $H_{s}^{1}\left(\Omega_{s}\right)$ and $H_{s}^{1}\left(\Omega_{s}\right)$ be a functional space in which we are searching the solution in accordance with its regularity $H_{s}^{1}=\left\{\phi^{k} \in H^{1}\left(\Omega_{s}\right) \mid \phi^{k}=s \forall x \in \Gamma_{s}\right\}, H_{s}^{1}=\left\{\psi^{k} \in H^{1}\left(\Omega_{g}\right) \mid \psi^{k}=s \forall x \in \Gamma_{g}\right\}$ and $H_{s}^{1}=\left\{\chi^{k} \in\right.$ $\left.H^{1}\left(\Omega_{g}\right) \mid \chi^{k}=s \forall x \in \Gamma_{s}\right\}$ where $H^{1}\left(\Omega_{s}\right)$ and $H^{1}\left(\Omega_{g}\right)$ are a Sobolev space, classicaly defined as $H^{1}\left(\Omega_{s}\right)=\left\{\phi^{k} \in\right.$ $\left.L^{2}\left(\Omega_{s}\right),\left\|\nabla \phi^{k}\right\| \in L^{2}\left(\Omega_{s}\right)\right\}, H^{1}\left(\Omega_{g}\right)=\left\{\phi^{k} \in L^{2}\left(\Omega_{g}\right),\left\|\nabla \phi^{k}\right\| \in L^{2}\left(\Omega_{g}\right)\right\}$ and $H^{1}\left(\Omega_{s}\right)=\left\{\chi \in L^{2}\left(\Omega_{s}\right),\left\|\nabla \chi^{k}\right\| \in\right.$ $\left.L^{2}\left(\Omega_{s}\right)\right\}$. Where $L^{2}\left(\Omega_{s}\right)$ and $L^{2}\left(\Omega_{g}\right)$ are the Hilbert vector space of the functions quadrically summable respectively on $\left(\Omega_{s}\right)$ and $\left(\Omega_{g}\right)$. 
We have, $L^{2}\left(\Omega_{s}\right)=\left\{\left.\phi^{k}(x)\left|\int_{\Omega_{s}}\right| \phi^{k}(x)\right|^{2} d x<\infty\right\}, L^{2}\left(\Omega_{g}\right)=\left\{\left.\psi^{k}(x)\left|\int_{\Omega_{g}}\right| \psi^{k}(x)\right|^{2} d x<\infty\right\}$ and $L^{2}\left(\Omega_{s}\right)=$ $\left\{\left.\chi^{k}(x)\left|\int_{\Omega_{s}}\right| \chi^{k}(x)\right|^{2} d x<\infty\right\}$. The norm of these space are:

$\left\|\phi^{k}\right\|=\left(\int_{\Omega_{s}} \nabla \phi^{k} \cdot \nabla \phi^{k} d \mathbf{x}\right)^{\frac{1}{2}} ; \quad\left\|\psi^{k}\right\|=\left(\int_{\Omega_{g}} \nabla \psi^{k} \cdot \nabla \psi^{k} d \mathbf{x}\right)^{\frac{1}{2}} ; \quad\left\|\chi^{k}\right\|=\left(\int_{\Omega_{s}} \nabla \chi^{k} \cdot \nabla \chi^{k} d \mathbf{x}\right)^{\frac{1}{2}}$

By using the Green's theorem, integration by parts leads to,

$$
\left\{\begin{aligned}
\int_{\Omega_{s}} \frac{\partial C^{s}}{\partial t} \phi^{k} d V^{\Omega_{i}} & =-\int_{\Omega_{s}} \mathbf{D}_{s} \nabla C^{s} \cdot \nabla \phi^{k} d V^{\Omega_{i}}+\int_{\partial \Omega_{s}} \mathbf{D}_{s} \nabla C^{s} \cdot \mathbf{n} \phi^{k} d S^{\Omega_{i}}+\int_{\Omega_{s}} \mathbf{q}_{1} \phi^{k} d V^{\Omega_{i}} \\
\int_{\Omega_{g}} \frac{\partial C^{g}}{\partial t} \psi^{k} d V^{\Omega_{i}} & =-\int_{\Omega_{g}} \mathbf{D}_{g} \nabla C^{g} \cdot \nabla \psi^{k} d V^{\Omega_{i}}+\int_{\partial \Omega_{g}} \mathbf{D}_{g} \nabla C^{g} \cdot \mathbf{n} \psi^{k} d S^{\Omega_{i}}-\int_{\Omega_{g}} \nabla \cdot\left(\mathbf{u} C^{g}\right) \psi^{k} d V^{\Omega_{i}} \\
& +\int_{\Omega_{s}}^{\mathbf{q}_{2} \psi^{k} d V^{\Omega_{i}}} \\
\rho^{s} c^{s} \int_{\Omega_{s}} \frac{\partial T^{s}}{\partial t} \chi^{k} d V^{\Omega_{i}} & =-\int_{\Omega_{s}} \kappa_{s} \nabla T^{s} \cdot \nabla \chi^{k} d V^{\Omega_{i}}+\int_{\partial \Omega_{s}} \kappa_{s} \nabla T^{s} \cdot \mathbf{n} \chi^{k} d S^{\Omega_{i}}-\int_{\Omega_{g}} \nabla \cdot\left(\mathbf{u} T^{s}\right) \chi^{k} d V^{\Omega_{i}} \\
& +\int_{\Omega_{s}} \rho^{s} \mathbf{r} \chi^{k} d V^{\Omega_{i}}
\end{aligned}\right.
$$

By applying the Galerkin weighted residual methods and the Green's theorem, the variational formulation corresponding the AMCs cross contamination is given by the equation (1). We now introduce the weak formulation of the AMCs model:

We find $C^{s} \in H^{1}\left(\Omega_{s}\right)$ and $C^{g} \in H^{1}\left(\Omega_{g}\right)$ such that,

$$
\left\{\begin{array}{lll}
a_{1}^{k}\left(\frac{\partial C^{s}}{\partial t}, \phi^{k}\right)+b_{1}^{k}\left(C^{s}, \phi^{k}\right) & =L_{1}^{k}\left(\phi^{k}\right) & \forall \phi^{k} \in H^{1}\left(\Omega_{s}\right) \\
a_{2}^{k}\left(\frac{\partial C^{g}}{\partial t}, \psi^{k}\right)+b_{2}^{k}\left(C^{g}, \psi^{k}\right)=L_{2}^{k}\left(\psi^{k}\right) & \forall \psi^{k} \in H^{1}\left(\Omega_{g}\right) \\
a_{3}^{k}\left(\frac{\partial T^{s}}{\partial t}, \chi^{k}\right)+b_{3}^{k}\left(T^{s}, \chi^{k}\right)=L_{3}^{k}\left(\chi^{k}\right) & \forall \chi^{k} \in H^{1}\left(\Omega_{s}\right)
\end{array}\right.
$$

where

$$
\begin{aligned}
& L_{1}^{k}\left(\phi^{k}\right)=\int_{\Omega_{s}} \mathbf{q}_{1} \phi^{k} d V^{\Omega_{i}} ; \quad L_{2}^{k}\left(\psi^{k}\right)=\int_{\Omega_{g}} \mathbf{q}_{2} \psi^{k} d V^{\Omega_{i}}+\int_{\Omega_{g}} N_{0}^{g} \psi^{k} d S^{\Omega_{i}} ; \quad L_{3}^{k}\left(\chi^{k}\right)=\int_{\Omega_{s}} \rho^{s} \mathbf{r} \chi^{k} d V^{\Omega_{i}} ; \\
& a_{1}^{k}\left(\frac{\partial C^{s}}{\partial t}, \phi^{k}\right)=\int_{\Omega_{s}} \frac{\partial C^{s}}{\partial t} \phi^{k} d V^{\Omega_{i}} ; \quad a_{2}^{k}\left(\frac{\partial C^{g}}{\partial t}, \psi^{k}\right)=\int_{\Omega_{g}} \frac{\partial C^{g}}{\partial t} \psi^{k} d V^{\Omega_{i}} ; \quad a_{3}^{k}\left(\frac{\partial T^{s}}{\partial t}, \chi^{k}\right)=\int_{\Omega_{s}} \rho^{s} c^{s} \frac{\partial T^{s}}{\partial t} \chi^{k} d V^{\Omega_{i}} \\
& b_{1}^{k}\left(C^{s}, \phi^{k}\right)=\int_{\Omega_{s}} \mathbf{D}_{s} \nabla C^{s} \cdot \nabla \phi^{k} d V^{\Omega_{i}}+\int_{\Gamma_{N}} h_{0} C^{g} \phi^{k} d S^{\Omega_{i}} \\
& b_{2}^{k}\left(C^{g}, \psi^{k}\right)=\int_{\Omega_{g}}^{\Omega_{s}} \mathbf{D}_{g} \nabla C^{g} \cdot \nabla \psi^{k} d V^{\Omega_{i}}+\int_{\Omega_{g}}^{\Gamma_{N}} \nabla \cdot\left(\mathbf{u} C^{g}\right) \psi^{k} d V^{\Omega_{i}}+\int_{\Gamma_{N}} \frac{C^{s}}{h_{0}} \psi^{k} d S^{\Omega_{i}}+\int_{\Gamma_{D}} k_{c}\left[C_{0} H(t-\varepsilon)-C^{g}\right] \psi^{k} d S^{\Omega_{i}} \\
& b_{3}^{k}\left(T^{s}, \chi^{k}\right)=\int_{\Omega_{g}} \kappa_{s} \nabla T^{s} \cdot \nabla \chi^{k} d V^{\Omega_{i}}+\int_{\Omega_{s}} \nabla \cdot\left(\mathbf{u} T^{s}\right) \chi^{k} d V^{\Omega_{i}}
\end{aligned}
$$

The domain $\Omega_{s}$ and $\Omega_{g}$ are decomposed in a finite number of subdomains $\Omega_{s}^{e}$ and $\Omega_{g}^{e}$ for each elements. Similarly, the boundary $\partial \Omega_{s}$ and $\partial \Omega_{g}$ are decomposed in $\partial \Omega_{s}^{e}$ and $\partial \Omega_{g}^{e}$. Finally the time interval is subdivised by $n$ subinterval. For the spatial discretization, we assume the finite element partition $\mathcal{T}_{h}^{s}$ and $\mathcal{T}_{h}^{s}$ of $\Omega_{s}$ and $\Omega_{g}$ respectively into tetrahedral elements. Again for simplicity, we will assume that the finite element partition associated to $\mathcal{T}_{h}^{s}$ and $\mathcal{T}_{h}^{s}$ are uniform, $h$ is the size of the element domains. Let us $C_{h}^{s}$ and $C_{h}^{g}$ de the approximation solution of $C_{h}^{s}$ and $C_{h}^{g}$ respectively.

The Galerkin approximation above became:

Find $C_{h}^{s} \in H^{1 h}\left(\Omega_{g}\right)$ and $C_{h}^{g} \in H^{1 h}\left(\Omega_{g}\right)$ such that,

$$
\left\{\begin{array}{l}
a_{1}^{k}\left(\frac{\partial C_{h}^{s}}{\partial t}, \phi_{h}^{k}\right)+b_{1}^{k}\left(C_{h}^{s}, \phi_{h}^{k}\right)=L_{1}^{k}\left(\phi_{h}^{k}\right) \quad \forall \phi_{h}^{k} \in H^{1 h}\left(\Omega_{s}\right) \\
a_{2}^{k}\left(\frac{\partial C_{h}^{g}}{\partial t}, \psi_{h}^{k}\right)+b_{2}^{k}\left(C^{g}, \psi_{h}^{k}\right)=L_{2}^{k}\left(\psi_{h}^{k}\right) \quad \forall \phi_{h}^{k} \in H^{1 h}\left(\Omega_{g}\right) \\
a_{3}^{k}\left(\frac{\partial T_{h}^{s}}{\partial t}, \chi_{h}^{k}\right)+b_{3}^{k}\left(T^{s}, \psi_{h}^{k}\right)=L_{3}^{k}\left(\chi_{h}^{k}\right) \quad \forall \chi_{h}^{k} \in H^{1 h}\left(\Omega_{s}\right)
\end{array}\right.
$$


Finally, we have a system of first order differential equations and using the matricial notation we have, Where the appropriate test function respectively $\phi, \psi$ and $\chi$ for the concentration $C^{s} \in \mathbb{R}^{2}, C^{g} \in \mathbb{R}^{2}$ and the temperature $T^{s} \in \mathbb{R}^{2}$ respectively. The discretization is similar with the discretization defined in section (3).

According to the equation (7) defined at section (3) of this work, we have to solve:

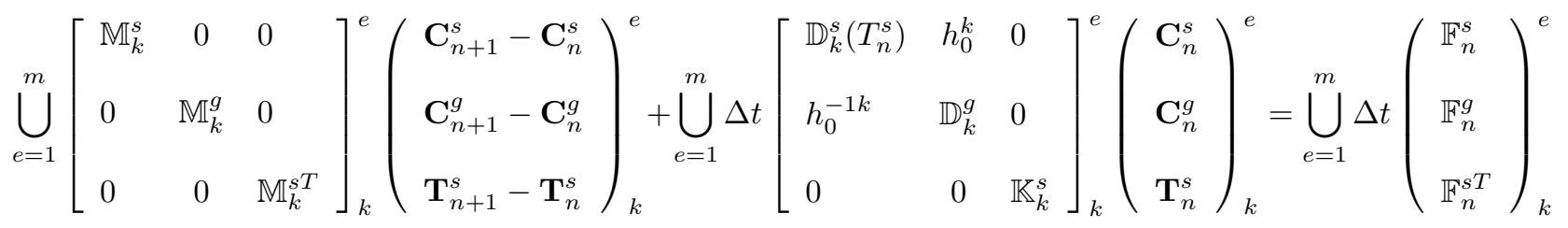

Where

$$
\begin{array}{r}
\mathbb{F}_{n}^{s}=\int_{\Omega_{s}^{e}} \mathbf{q}_{1} \mathbf{N} d V^{\Omega_{i}} ; \quad \mathbb{F}_{n}^{g}=\int_{\Omega_{s}^{e}} \mathbf{q}_{1} \mathbf{N} d V^{\Omega_{i}}+\int_{\Omega_{g}^{e}} N_{0}^{g} \mathbf{N} d V^{\Omega_{i}} ; \quad \mathbb{F}_{n}^{s T}=\int_{\Omega_{s}^{e}} \rho^{s} \mathbf{r} \mathbf{N} d V^{\Omega_{i}} ; \quad \mathbb{M}_{k}^{s}=\int_{\Omega_{s}^{e}} \mathbf{N}^{\mathbf{T}} \mathbf{N} d V^{\Omega_{i}} ; \\
\mathbb{M}_{k}^{g}=\int_{\Omega_{g}^{e}} \mathbf{N}^{\mathbf{T}} \mathbf{N} d V^{\Omega_{i}} ; \quad \mathbb{M}_{k}^{s T}=\int_{\Omega_{g}^{e}} \rho^{s} c^{s} \mathbf{N}^{\mathbf{T}} \mathbf{N} d V^{\Omega_{i}} ; \quad \mathbb{D}_{k}^{s}=\int_{\Omega_{s}^{e}} \mathbf{D}_{s}(T) \nabla \mathbf{N}^{\mathbf{T}} \nabla \mathbf{N} d V^{\Omega_{i}}+\int_{\Gamma_{N}} h_{0} \mathbf{N}^{\mathbf{T}} \mathbf{N} d S^{\Omega_{i}} ; \\
\mathbb{D}_{k}^{g}=\int_{\Omega_{g}^{e}} \mathbf{D}_{g}(T) \nabla \mathbf{N}^{\mathbf{T}} \cdot \nabla \mathbf{N} d V^{\Omega_{i}}+\int_{\Omega_{g}^{e}} \nabla \cdot\left(\mathbf{u N}^{\mathbf{T}}\right) \mathbf{N} d V^{\Omega_{i}}+\int_{\Gamma_{N}} \frac{\mathbf{N}^{\mathbf{T}}}{h_{0}} \mathbf{N} d S^{\Omega_{i}}+\int_{\Gamma_{D}} k_{c}\left[C_{0} H(t-\varepsilon)-\mathbf{N}^{\mathbf{T}}\right] \mathbf{N} d S^{\Omega_{i}} ; \\
\mathbb{K}_{k}^{s}=\int_{\Omega_{g}^{e}} \kappa_{s} \nabla \mathbf{N}^{\mathbf{T}} \cdot \nabla \mathbf{N} d V^{\Omega_{i}}+\int_{\Omega_{g}^{e}} \nabla \cdot\left(\mathbf{u} \mathbf{N}^{\mathbf{T}}\right) \mathbf{N} d V^{\Omega_{i}}
\end{array}
$$

and $\mathbf{N}$ denote the linear interpolation function at each node.

And by using the assembly theory for all subdomain, we have:

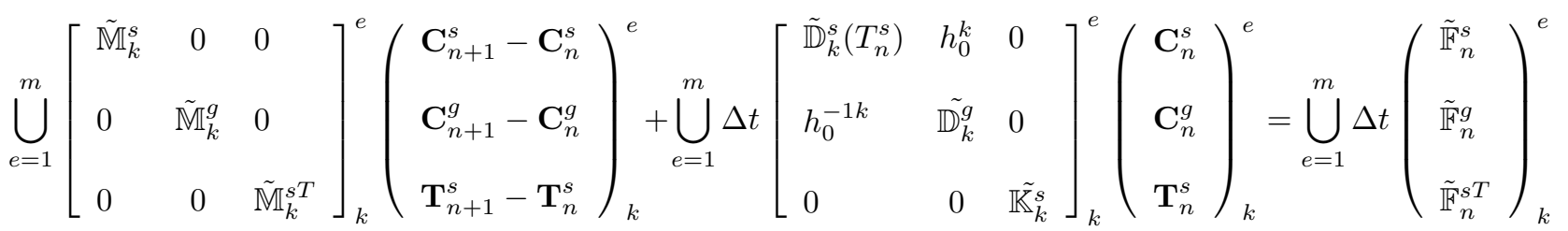

Where

$$
\begin{array}{rlrl}
\left(\tilde{\mathbb{M}}_{k}^{s, g}\right)_{i j} & =\sum_{i, j}^{p_{\text {nodes }}}\left(\mathbb{M}_{k}^{s, g}\right)_{i j} ; & \left(\tilde{\mathbb{D}}_{k}^{s, g}\right)_{i j}=\sum_{i, j}^{p_{\text {nodes }}}\left(\mathbb{D}_{k}^{s, g}\right)_{i j} ; & \left(\tilde{\mathbb{F}}_{k}^{s, g}\right)_{j}=\sum_{j}^{p_{\text {nodes }}}\left(\mathbb{F}_{k}^{s, g}\right)_{j} ; \\
\left(\tilde{\mathbb{M}}_{k}^{s T}\right)_{i j}=\sum_{i, j}^{p_{\text {nodes }}}\left(\mathbb{M}_{k}^{s T}\right)_{i j} ; & \left(\tilde{\mathbb{K}}_{k}^{s}\right)_{i j}=\sum_{i, j}^{p_{\text {nodes }}}\left(\mathbb{K}_{k}^{s}\right)_{i j} ; & \left(\tilde{\mathbb{F}}_{k}^{s T}\right)_{j}=\sum_{j}^{p_{\text {nodes }}}\left(\mathbb{F}_{k}^{s T}\right)_{j} ;
\end{array}
$$

\section{Applications in industrial processes}

\subsection{Contamination process}

This process illustrates the process during which the wafer post processed outgass the contaminant and contamined the internal surface FOUP. This is the first step of the cross contamination. The contamination moves from wafer to FOUPs. During this process, the surface adsorption step take place and the diffusion in the volume of the FOUP happened. Contamination process consist to use the same condition in section (3) of this work, in which we consider that the wafer is the contaminant source governed by the Heaviside function with a delay $\varepsilon$. The implementation of this boundary condition is defined in equation (4). In fact, during $t_{c} \in \mathbb{R}_{+}$(contamination time), we apply on $\Gamma_{D}$, the condition can expressed as:

$$
\left\{\begin{array}{llr}
C^{g} & =C_{0} H(t-\varepsilon) & \text { if } 0<t \leq \tilde{t}_{c} \\
\left(-\mathbf{D}_{g} \nabla C^{g}+\mathbf{u} C^{g}\right) \cdot \mathbf{n}= & 0 & \text { if } \tilde{t}_{c}<t \leq\left(\tilde{t}_{c}+t_{o}\right)
\end{array}\right.
$$


The experimental process prescribe that the contamination time $t_{c} \in \mathbb{R}_{+}$is decomposed in two characteristic time $\tilde{t}_{c} \in \mathbb{R}_{+}$and $t_{o} \in \mathbb{R}_{+}$respectively the time until the wafer outgass is finished and the waiting time before opening the FOUP to remove the wafer.

For the implementation of the boundary conditions also defined in the equation (52), we use the same method with the switch condition using the inflow concentration flux. We run this test with many kind of material and with different level of initial concentration $C_{0}$.

\subsection{Outgassing process}

Outgassing process take place after opening the FOUP in the goal to remove the wafer. The FOUP's atmosphere change and the contaminant concentration $C^{g} \simeq 0$ during the operation, after $t_{p} \in \mathbb{R}_{+}$the front door will be close. This step results a reverse flow of the contamination gradient during which the contaminant moves from FOUP to wafer. After the intentional contamination of the FOUP during the contamination $t_{c} \in \mathbb{R}_{+}$, just after $t_{c} \in \mathbb{R}_{+}$the FOUP has been opened during the opening time $t_{p} \in \mathbb{R}_{+}$then the FOUP has been closed during the outgassing time $t_{d} \in \mathbb{R}_{+}$.

$$
\left\{\begin{array}{lllr}
C^{g} & = & 0 & \text { if } t_{c}<t \leq\left(t_{c}+t_{p}\right) \\
\left(-\mathbf{D}_{g} \nabla C^{g}+\mathbf{u} C^{g}\right) \cdot \mathbf{n} & = & 0 & \text { if }\left(t_{c}+t_{p}\right)<t \leq\left(t_{c}+t_{p}+t_{d}\right)
\end{array}\right.
$$

\subsection{Decontamination process}

In this section, we describe the application of the model in decontamination process of the pods. After the outgassing process, the decotamination begin. Decontamination is used to remove the contaminant already accumulated at the internal surface and diffused in the FOUP's material. Decontamination is an operation used of reducing the AMCs cross contamination risk by purging methods. In this work, we assume two types of these methods, the cold purging and the hot purging. In fact, we denote these two types of decontamination by cold decontamination and hot decontamination. In this section, many process of purge are assumed and are studied by theoretical analysis and are validate with the experimental measurement. The goal of this section is to study the temperature effect during the FOUPs cleaning. Purging FOUP with inert gas is the one of the most popular method, but there are many several method as UV or vacuum methods. In general purging the FOUP with inert gas provides the many advantages. The decontamination process will be done during $t_{u} \in \mathbb{R}_{+}$the decontamination duration and we denote $t_{w} \in \mathbb{R}_{+}$the waiting time when the FOUP is closed after decontamination process. This waiting process is important to known the cleaning efficacy. So the final time $T \in \mathbb{R}_{+}$of the simulation is the sum of all characteristic time $t_{i} \in \mathbb{R}_{+}$, it can be written:

$T=\sum_{i} t_{i}=t_{c}+t_{p}+t_{d}+t_{u}+t_{w}$

Ended, the total time is defined as $t \in\left[0, \sum_{i} t_{i}\right]$ or $t \in[0, T]$

\subsubsection{Cold decontamination}

We compute in this case the following boundary conditions given by the equation (55). There is no change in diffusion coefficient. We assume, in this section that the initial condition is the step phase before (after, removing wafer, outgassing for equilibrium atmosphere).

$$
\left\{\begin{array}{lll}
C^{g} & = & 0 \\
& \text { if }\left(t_{c}+t_{p}+t_{d}\right)<t \leq \sum_{i} t_{i}-t_{w} \\
\left(-\mathbf{D}_{g} \nabla C^{g}+\mathbf{u} C^{g}\right) \cdot \mathbf{n}= & 0 & \text { if } t>\sum_{i} t_{i}-t_{w}
\end{array}\right.
$$

\subsubsection{Hot decontamination}

We compute in this case same boundary condition the boundary conditions using the switch condition. The diffusion coefficient is function of the temperature. We assume, in this section that the initial condition is the step phase before (after, removing wafer, outgassing for equilibrium atmosphere).

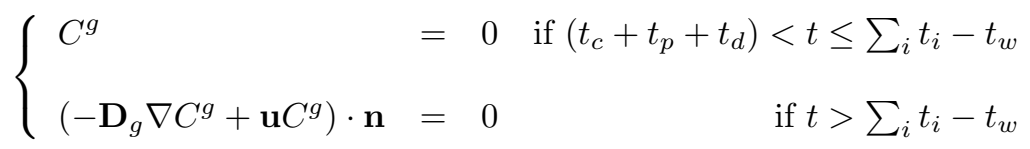


Where $t_{c}=\tilde{t}_{c}+t_{a}$, in which $\tilde{t}_{c} \in \mathbb{R}_{+}$is the contamination time (outgassing time from wafer to atmosphere and FOUP $), t_{a} \in \mathbb{R}_{+}$is the waiting time before opening, in this time the wafer outgass is already finished.

\section{Some results and discussion}

This section provides with illustrations and discussion some results of the computed model with its correspondence to the industrial applications.
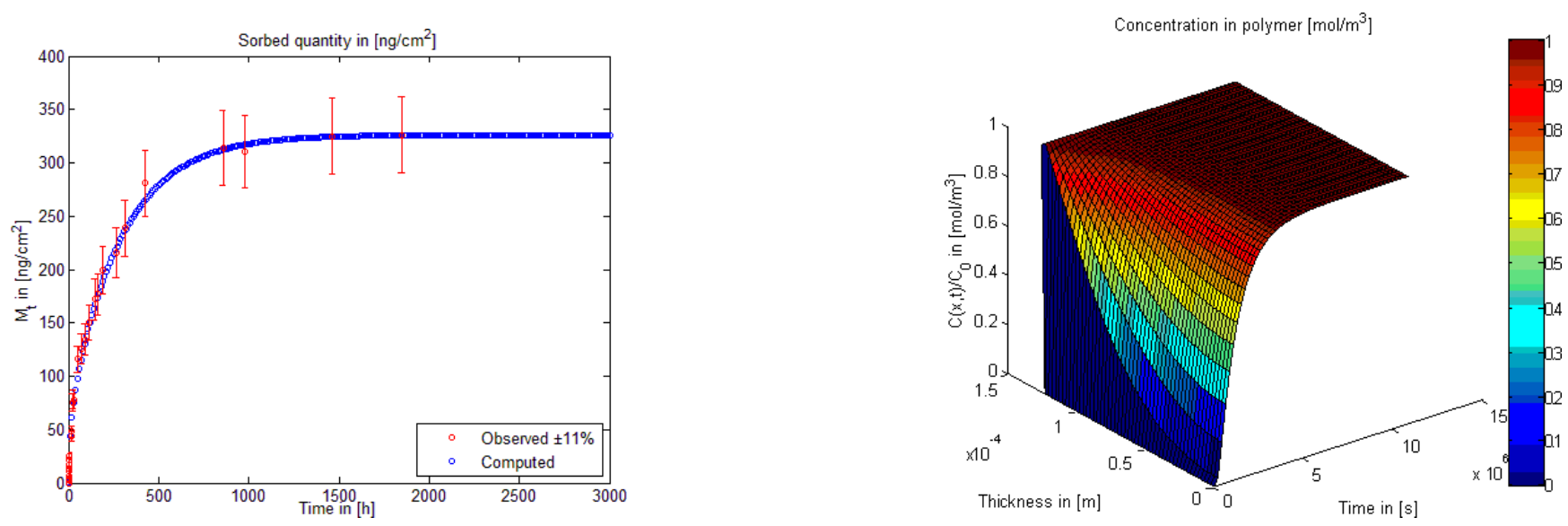

Figure 3: Sorbed quantity in the polymer in function of time for the contaminant $X C 1$ : Computed model in blue, experimental measurement in red (left). Sorbed quantity in the polymer in function of time and space for the contaminant $X C 1$ : Computed model (right).
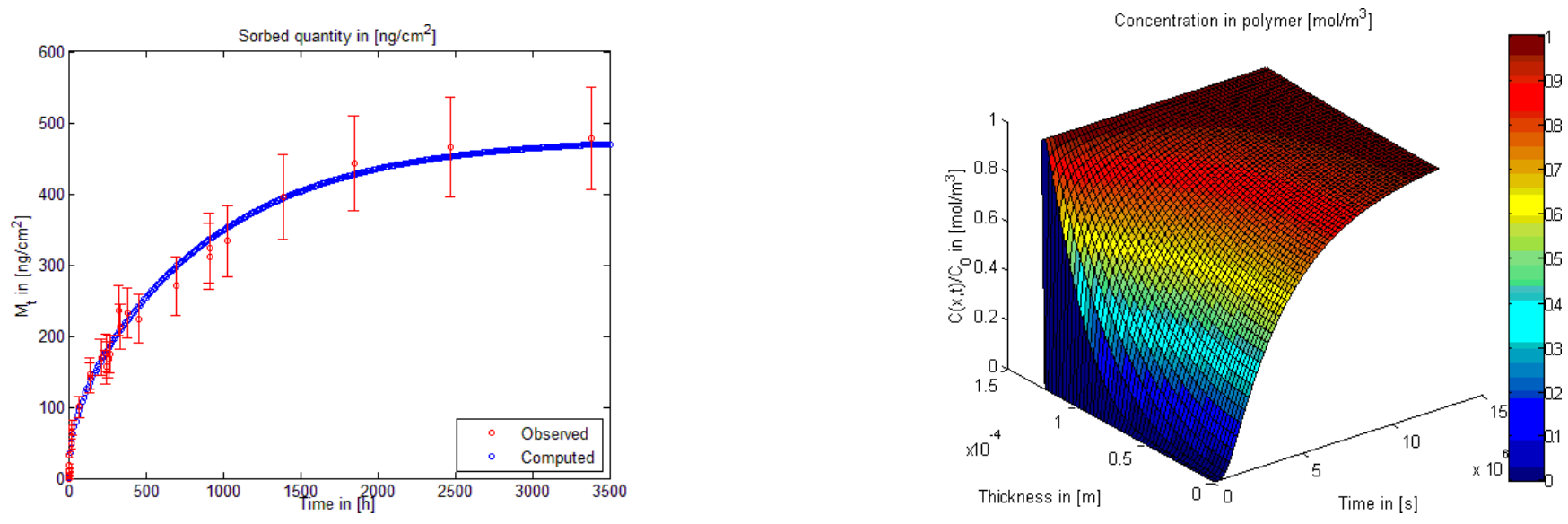

Figure 4: Sorbed quantity in the polymer in function of time for the contaminant $X C 2$ : Computed model in blue, experimental measurement in red (left). Sorbed quantity in the polymer in function of time and space for the contaminant $X C 2$ : Computed model (right).

The figures fig.3, illustrate the correlation between the computed model and the experimental measurement during the contamination process. The figures fig. 4 illustrates the correlation between the computed model and the experimental measurement during the contamination process. We can see that the mathematical model is in correlation with the experimental measurement. We use the first model given by the equation (3) to study the correlation of the model with the experimental measurement. We measured the sorbed quantity of the contaminant in the polymer with the IC method, and we obtained the diffusion coefficient with numerical optimisation by using the model.

The figure fig.5 illustrate the concentration in the polymer during $1[\mathrm{~h}]$ of contamination and $1[\mathrm{~h}]$ of waiting time. This result is obtained with the model without heat effect. This model and the results are in correlation with the experimental measurement, during the contamination process. We can see that we have two parts of the concentration gradient: the first parts are during the contamination process and the second part is during 

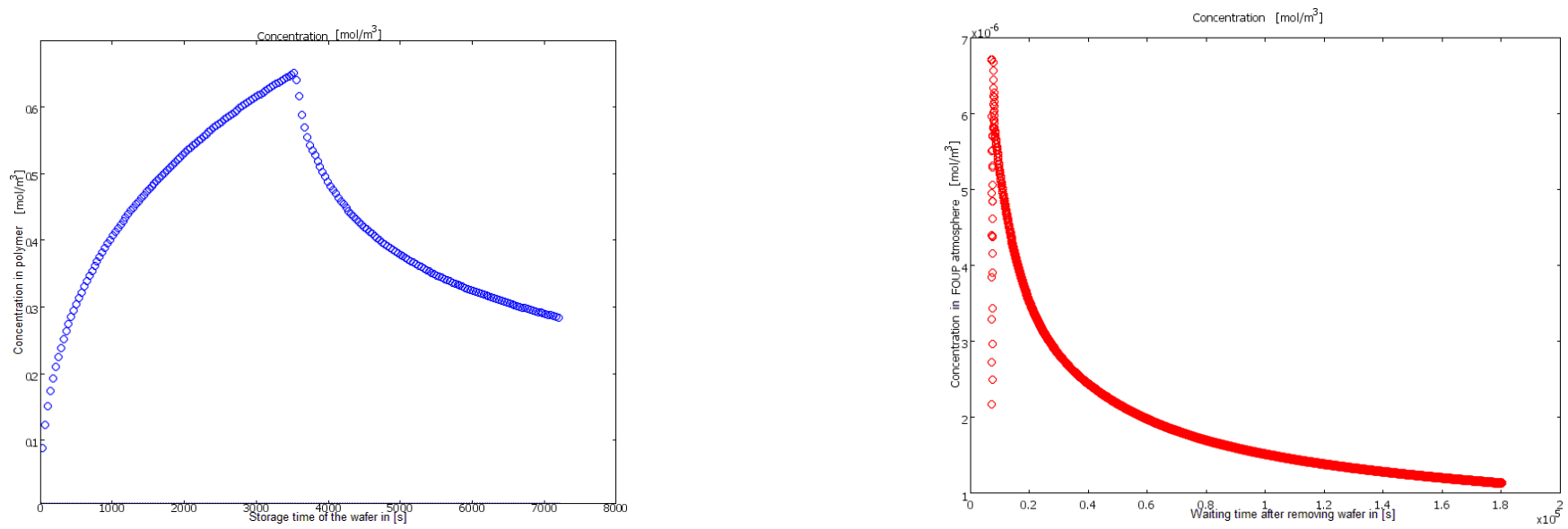

Figure 5: Contamination process: $1[\mathrm{~h}]$ of contamination and $1[\mathrm{~h}]$ of waiting time. The curve illustrate the concentration of the contaminant in the polymer in function of time (left). Outgassing: $5[\mathrm{mn}]$ removing wafer, 22[h] waiting time for atmosphere concentration equilibre. The curve illustrate the concentration of the contaminant in the FOUP's atmosphere in function of time (right).
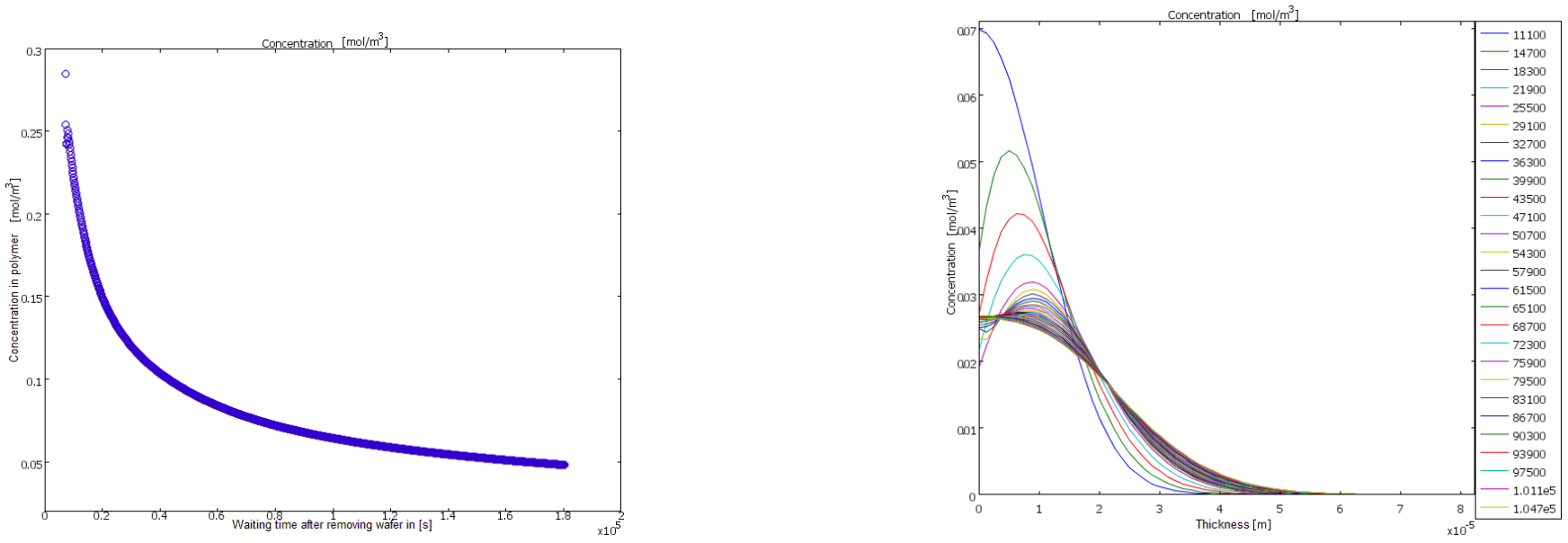

Figure 6: Outgassing: $5[\mathrm{mn}]$ removing wafer, $22[\mathrm{~h}]$ waiting time for atmosphere concentration equilibre. The curve illustrate the concentration of the contaminant in the polymer in function of time (left). Decontamination: the curve illustrates the concentration in the polymer in function of the space after decontamination. The computed results is obtained by using the model without heat effect (right).

the waiting time. We use the first model without the temperature effect given by the equation (3) to study this process. Thefig. 6 represents respectively the concentration in the FOUP's atmosphere and the polymer after $5[\mathrm{mn}]$ of FOUP's opening and $22[\mathrm{~h}]$ of waiting time. The step before is the contamination process give by the figure fig.5.

The figures fig.7, fig.8, and fig.9 illustrate the concentration in the polymer after decontamination of the FOUP. We can see that we have two parts of the concentration during the decontamination, the first part continue to diffuse in the polymer and the second part return back to the FOUP's atmosphere and contamines the new wafer. The dynamic of the AMCs cross contamination is determined by this phenomenon (adsorption and desorption properties of this material). The new wafer is contamined by the contaminant already adsorb in the polymer but with the effect of decontamination illustrated in the figure fig.9 the contamination return back to the FOUP's atmosphere. The figures fig.9 illustrates respectively the concentration in the polymer and in the FOUP's atmosphere during the FOUP's cleaning or decontamination. The figures fig.10 illustrates the concentration in function of time and space during the ideal desorption. During the ideal desorption we assume that there is no residual contaminant adsorbed in the polymer after decontamination process; all of the adsorbed contaminant will be desorbed. 

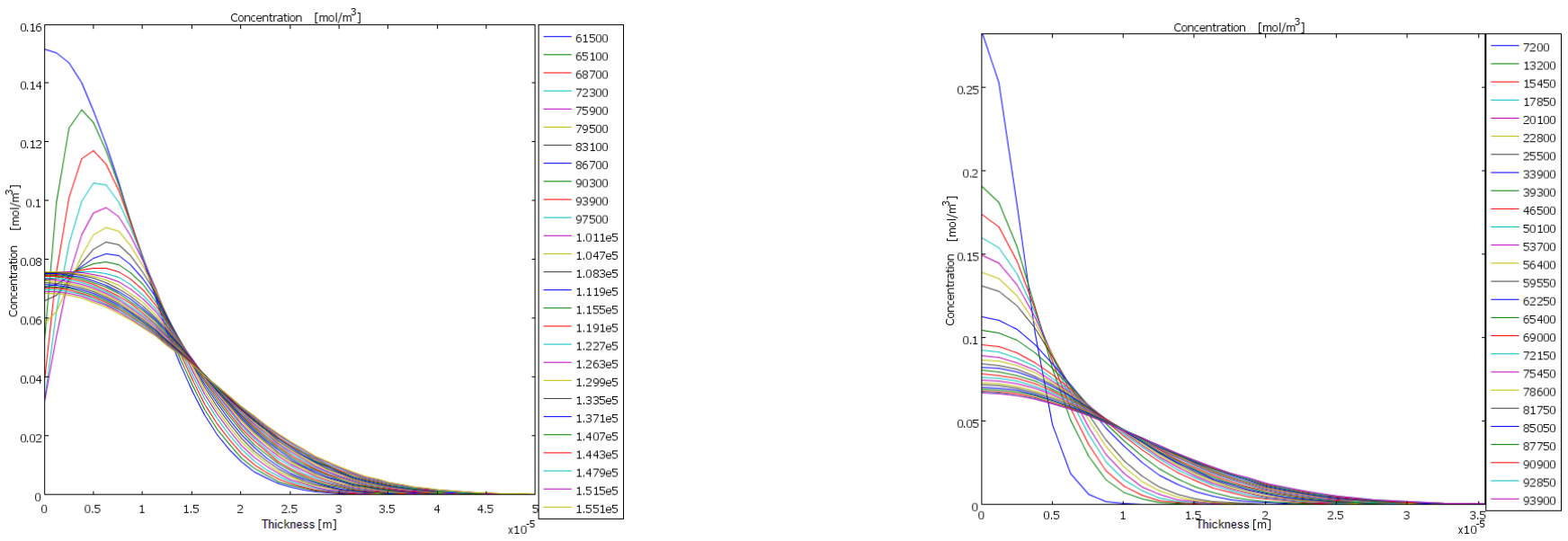

Figure 7: Decontamination: the curve illustrates the concentration in the polymer in function of the space after decontamination. The computed results is obtained by using the model without heat effect (left). Contamination: the curve illustrates the concentration in the polymer in function of the space after decontamination. The computed results is obtained by using the model without heat effect (right).
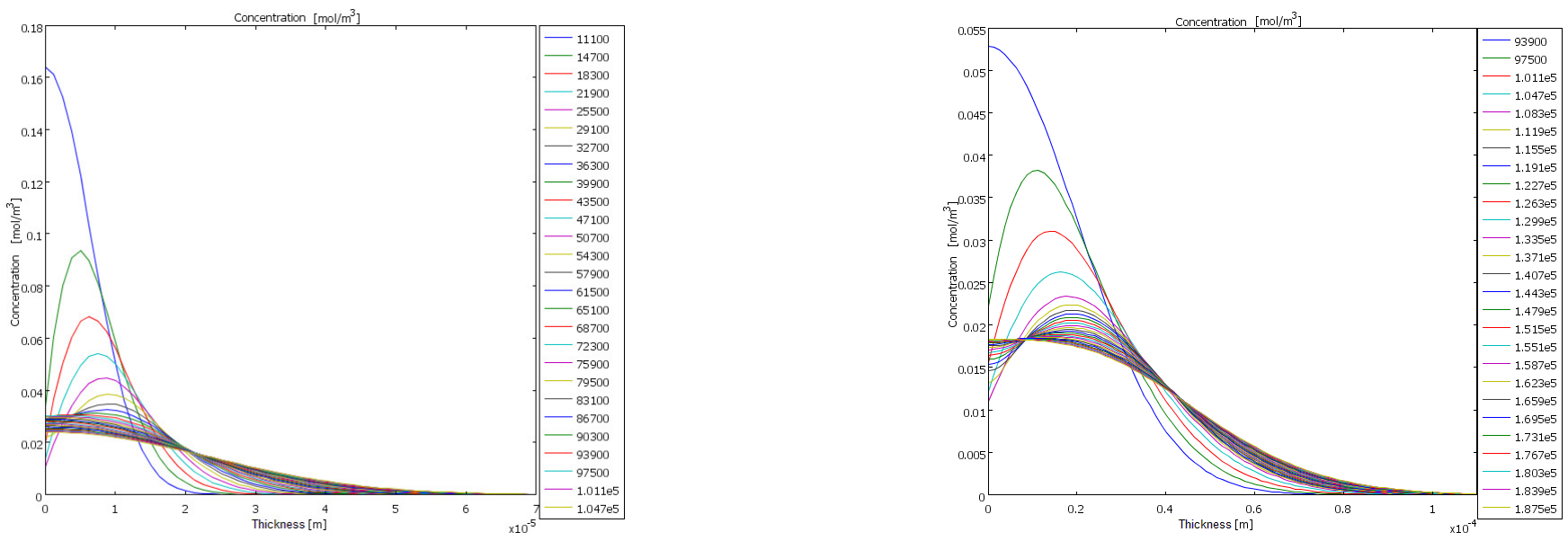

Figure 8: Decontamination: the curve illustrates the concentration in the polymer in function of the space after decontamination. The computed results is obtained by using the model with heat effect (left). Decontamination: the curve illustrates the concentration in the polymer in function of the space after decontamination. The computed results is obtained by using the model with heat effect (right).

\section{Concluding remarks}

The ultimate aim of this work is to understand and to quantify by using the modelling and simulation method a mathematical model with the temperature effect on the AMCs cross contamination. Then, the second aims are to predict with the modelling and simulation the behaviour of the contaminant in each material constituent of the FOUP. This method is used to study the sensitivity of each material constituent of the FOUP with a given contaminant. After the study of the behaviour, the one of the main objective of this work is to study the decontamination process with/without heat (temperature) effect. Mathematical model and numerical method are established. It is developed to predict the transient reversible and irreversible diffusion in the FOUP's polymer.

We can also see the temperature effect in the computed results that we have a benefit effects during decontamination process with the use of the hot decontamination. The use of the hot decontamination cleans maximum amount of contaminant in the polymer (support of the wafer and the body). One of the disadvantages of the hot decontamination is that it promotes the diffusion of the contaminant in the polymer (body) because the diffusion increases proportionally with the temperature. The used of the model with heat effect using the Arrhenius's law is the most benefit than using the model with the heat equation. The results are so similar but the last model has many calculations number for the computation. 

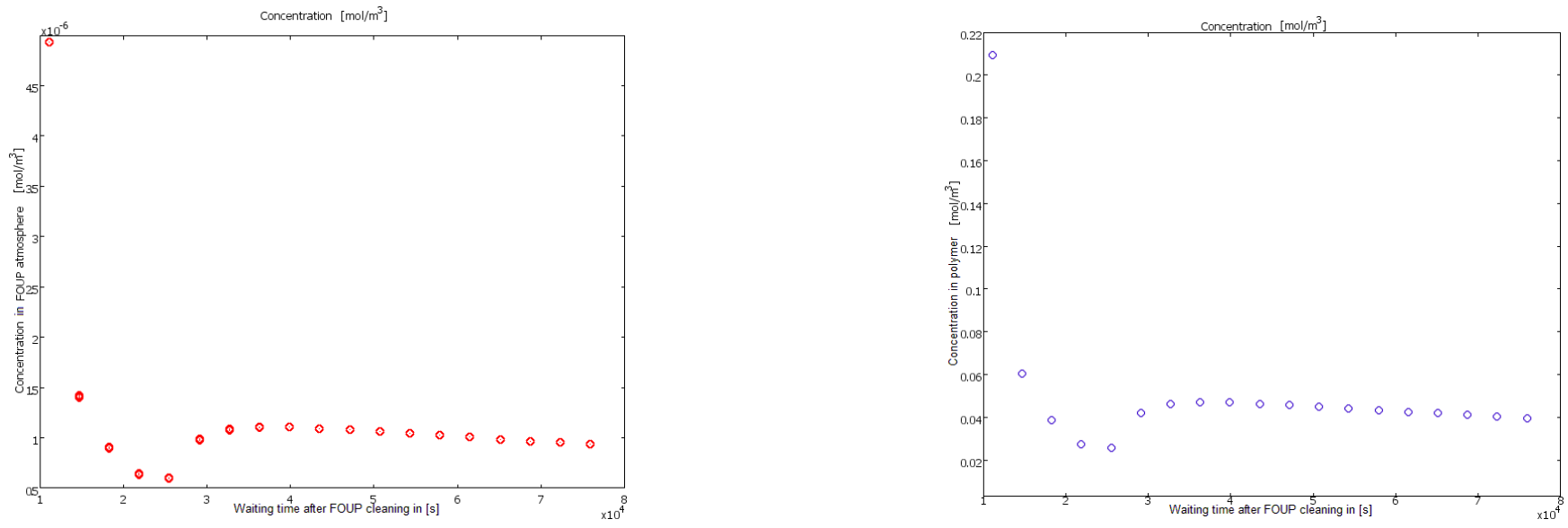

Figure 9: Cleaning process: $4[\mathrm{~h}]$ of cleanning time and $22[\mathrm{~h}]$ of waiting time. The curve represent the concentration in the FOUP's atmosphere in function of time (left). Cleaning process: $4[\mathrm{~h}]$ of cleanning time and $22[\mathrm{~h}]$ of waiting time. The curve represent the concentration in the polymer in function of time (right).
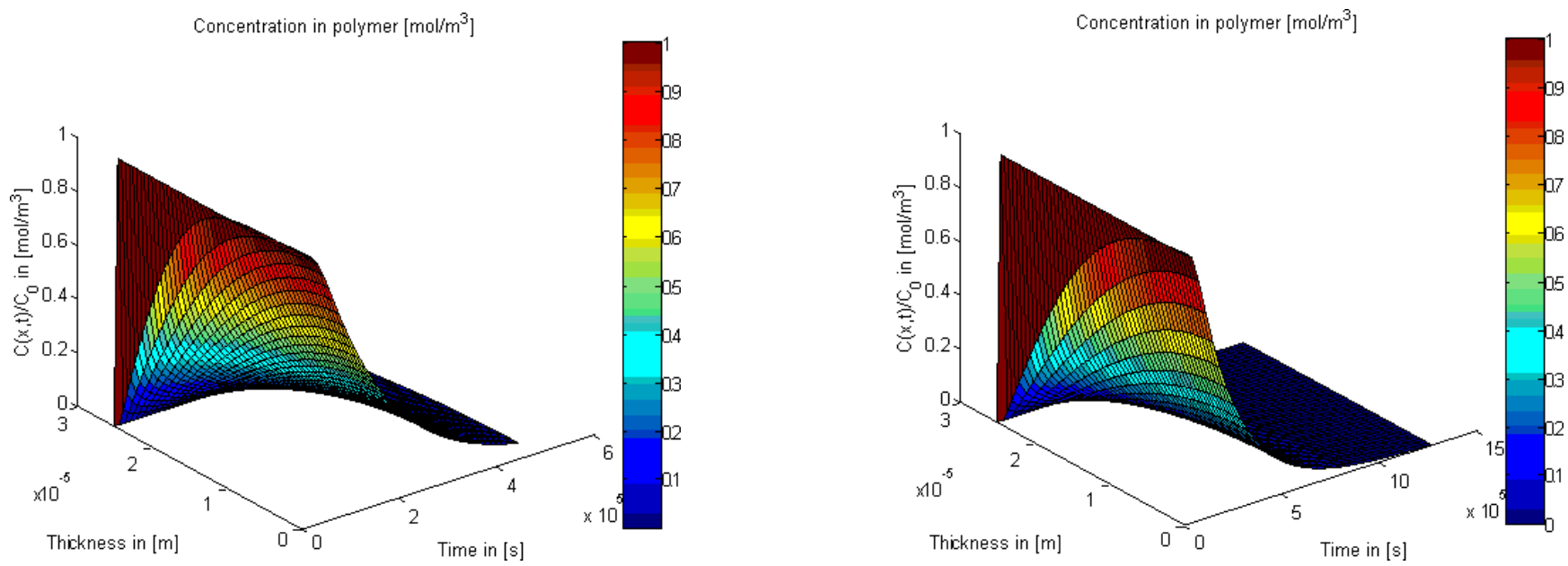

Figure 10: Desorbed (ideal desorption) process for the contaminant $X C 1$. The curve represent the concentration in function of the space and the time in the polymer (left). Desorbed (ideal desorption) process for the contaminant $X C 2$. The curve represent the concentration in function of the space and the time in the polymer (right).

The recontamination process is necessary to evaluate and quantify the AMCs cross contamination cycle after cleaning in the FOUP already used. During each cycle, contaminations, purge, cleaning processes another amount of contaminant may be add in the residual contaminant already adsorbed in the FOUP. A theoretical of this additional problem is required for example including the adsorption kinetics and the deposition kinetics. The present analysis has been formulated with all of the processes used in industry applications. The model is in correlation with the experimental measurement. This methodology is relevant for the industrial applications in cross-contamination control, cleaning control of the FOUP, we are focused on the dynamics of cross-contamination mainly on the evolution of the concentration level and the profile through the polymeric material, and finally on the effect of the material.

\section{References}

[1] T.Q.Nguyen, H.Fontaine and al. Identification and quantification of FOUP molecular contaminants inducing defects in integrated circuits manufacturing, Microelectronic Engineering, Vol. 105, (2013), pp. 124-129.

[2] P.Gonzàlez, H.Fontaine, C.Beitia and al. A comparative study of the HF sorption and outgassing ability of different Entegris FOUP platforms and materials, Microelectronic Engineering, Vol. 150, (2013), pp. 113-118. 
[3] S.Hu, T.Wu, and al. Design and evaluation of a nitrogen purge system for the front opening unifed pod, Applied Thermal Engineering, Vol. 27, (2007), pp. 1386-1393.

[4] H.Fontaine, H.Feldis and al. Impact of the volatile Acid Contaminant on Copper Interconnects, Electrical Perform, Vol. 25, No: 5, (2009), pp. 78-86.

[5] N. Santatriniaina, J.Deseure, T.Q.Nguyen, H.Fontaine, C. Beitia, L.Rakotomanana. Mathematical modeling of the AMCs cross-contamination removal in the FOUPs: Finite element formulation and application in FOUP's decontamination, Inter. Journ. of Math., comput. sci. engrg., Vol. 8, No: 4, (2014), pp. 409-414.

[6] N. Santatriniaina, J.Deseure, T.Q.Nguyen, H.Fontaine, C. Beitia, L.Rakotomanana. Coupled system of PDEs to predict the sensitivity of the some material constituents of the FOUP with the AMCs cross-contamination, International Journal of Applied Mathematical Research, Vol. 3, No: 3, (2014), pp. 233-243.

[7] Alemayeuhu Ambaw, Randolph Beaudry, Inge Bulens, Mulugeta Admasu Delele, Q.Tri Ho, Ann Schenk, Bart M. Nicolai, Pieter Verboven, Modelling the diffusion adsorption kinetics of 1-methylcyclopropene (1-MCP) in apple fruit and nontarget materials in storage rooms, Journal of Food Engineering, Vol. 102, (2011), pp. 257-265.

[8] J.A.Boscoboinik, S.J. Manzi, V.D.Pereyra Adsorption-desorption kinetics of monomer-dimer mixture, Physics A, Vol. 389, (2010), pp. 1317-1328.

[9] Rico F. Tabor, Julian Eastoe, Peter J. Dowding, A two-step model for surfactant adsorption at solid surfaces, Journal of Colloid and Interface Science, Vol. 346, (2010), pp. 424.428.

[10] Anli Geng, Kai-Chee Loh, Effects of adsorption kinetics and surface heterogeneity on band spreading in perfusion chromatography-a network model analysis, Chemical Engineering Science, Vol. 59, (2004), pp. 2447-2456.

[11] Hiroki Nagaoka and Toyoko Imae, Ananlytical investigation of two-step adsorption kinetics on surfaces, Journal of Colloid and Interface Science, Vol. 264, (2003), pp. 335-342.

[12] J. Crank, The mathematics of diffusion, second edition, 1975 Clarendon Press, Oxford.

[13] R.Hirsch, C.C.Muller-Goymann, Fitting of diffusion coefficients in a three compartement sustained release drug formulation using a genetic algoritm, International Journal of Pharmaceutics, Vol. 120, (1995), pp. 229-234.

[14] K.J.Kuijlaars, C.R.Kleijin, H.E.A. van den Akker, Multi-component diffusion phenomena in multiple-wafer chemical vapour deposition reactors, The chemical Engineering Journal, Vol. 57, (2009), pp. 127-136.

[15] Koichi Aoki, Diffusion-controlled current with memory, Journal of electroanalytical Chemistry, Vol. 592, (2006), pp. $31-36$.

[16] Shengping Ding, William T. Petuskey, Solutions to Ficks second law of diffusion with a sinusoidal excitation, Solide State Ionics, Vol. 109, (1998), pp. 101-110.

[17] Juergen Siepmann, Florence Siepmann, Modeling of diffusion controlled drug delivery, Journal of Controlled Release, Vol. 161, (2012), pp. 351-362.

[18] H.Denny Kamaruddin, William J.Koros,Some observation about the application of Fick's first law for membrane separation of multicomponent mixtures, Journal of Membrane Science, Vol. 1135, (1997), pp. 47-159.

[19] Ana Rita C. Duarte, Carlos Martins, Patricia Coimbra, Maria H.M. Gil, Herminio C. de Sousa, Catarina M.M. Duarte, Sorption and diffusion of dense carbon dioxide in a biocompatible polymer, Journal of Supercitical Fluids, Vol. 38, (2006), pp. 392-398.

[20] Wu Hai-jin, Lin Bai-quan, Yao Qian, The theory model and analytic answer of gas diffusion, Procedia Earth and Planetary Science, Vol. 1, (2009), pp. 328-335.

[21] Lagarias, J., Reeds, J., Wright, M., and Wright, Convergence Properties of the Nelder-Mead Simplex Method in Low Dimensions, P SIAM Journal on Optimization, Vol. 9, No: 1, (1998), pp. 12-147.

[22] Hervé Fontaine, H. Feldis, A. Danel, S. Cetre, C. Ailhas, Impact of the volatile Acid Contaminant on Copper Interconnects, Electrical Performances. ECS Transactions, Vol. 25, No: 5, (2009), pp. 78-86. 